\title{
ASYMPTOTIC ANALYSIS OF A BUCKLING PROBLEM FOR AN EMBEDDED SPHERICAL SHELL*
}

\author{
G. W. JONES ${ }^{\dagger}$, S. J. CHAPMAN ${ }^{\dagger}$, AND D. J. ALLWRIGHT ${ }^{\dagger}$
}

\begin{abstract}
The axisymmetric buckling of a spherical shell embedded in an elastic medium with uniaxial compression at infinity is examined in the limit of small shell thickness ratio. An asymptotic method is developed by considering the paradigm problem of a beam attached to a Winkler substrate of variable stiffness, which in the small aspect ratio limit displays the same behavior as the shell. The asymptotic method is then applied to the Euler-Lagrange equations corresponding to shell buckling. The system is analyzed in two distinguished limits, displaying good agreement with the full numerical results.
\end{abstract}

Key words. shell theory, asymptotic expansions, linear elasticity, WKB theory

AMS subject classifications. 41A60, 74B05, 74K25

DOI. $10.1137 / 080735114$

1. Introduction. The buckling of elastic structures has been studied for centuries, beginning with Euler's research on a buckling strut and encompassing later theories for rod, plate, and shell buckling. Of major importance to many applications is the study of how attachment to a substrate affects the buckling behavior of a thin structure. A recent article of ours [12] examined the buckling problem of a spherical shell embedded in a linearly elastic medium, which is subjected to a uniaxial compressive stress. This problem is intended to model the compression of a class of anechoic tiles for underwater structures, whose microstructure consists of a distribution of microspheres (spherical shells whose radii are typically $\sim 20 \mu \mathrm{m}$ ) embedded in a rubber matrix. Other composite materials having a similar structure, such as syntactic foams $[2,15,16]$, may also benefit from this analysis.

Restricting the shell buckling patterns to be axisymmetric, in [12] the lowest critical stress for buckling was found, together with the associated buckling pattern, which was confined to a band around the shell's equator. One of the drawbacks of the calculation method was that it was seminumerical, in that the buckling patterns were found as an infinite series whose coefficients had to be found by solving an infinite system of linear equations. Thus a simple relation between the critical buckling stress and the properties of the shell and medium was impossible. In this paper we hope to remedy this by taking advantage of the smallness of two parameters of the problem: specifically the thickness ratio of the shell and the ratio of shear moduli in the shell and medium. Numerical experiments show that the wavelength of the buckling pattern becomes ever smaller as these parameters tend to zero.

This is reminiscent of behavior seen in other structures that are attached to elastic substrates. The most familiar of these is the wrinkling of skin, but other situations displaying the same sort of behavior are train tracks attached to the ground, the

${ }^{*}$ Received by the editors September 11, 2008; accepted for publication (in revised form) June 2, 2009; published electronically August 12, 2009.

http://www.siam.org/journals/siap/70-3/73511.html

† Oxford Centre for Industrial and Applied Mathematics, Mathematical Institute, 24-29 St Giles', Oxford, OX1 3LB, United Kingdom (jonesg@maths.ox.ac.uk, chapman@maths.ox.ac.uk, allwrigh@ maths.ox.ac.uk). The work of the first author was supported by a CASE studentship, jointly funded by EPSRC and Dstl. The work of the first and second authors was partially supported by EPSRC as part of the "New Frontiers in the Mathematics of Solids" (OxMOS) programme EP/D048400/1.

901 
wrinkling of dried fruit, and the buckling of carbon nanotubes embedded in epoxy [7]. In all these cases, the buckling wavelength is determined by a balance between the bending stiffness of the thin structure and the mechanical stiffness of the substrate. A higher bending stiffness tends to lengthen the buckling wavelength while a stiffer substrate shortens it [3]. This general philosophy is seen in the shell problem as well, since the ratio of bending stiffness to substrate stiffness involves both parameters identified earlier. However, the exact functional relationship of the wavelength on the parameters is not readily identifiable. Our hope is that an appeal to a simpler problem can give rise to a method which will allow us to analyze the shell buckling problem asymptotically, yielding both a buckling pattern and a good estimate of the critical buckling stress.

Following a review of the setup and results of [12] in section 2, we consider in section 3 the paradigm problem of a beam attached to a substrate of variable stiffness. This has a similar buckling pattern to the shell problem and is thus an ideal choice to develop our method. Section 4 examines the behavior of an elastic halfspace subjected to oscillatory displacement boundary conditions, which will be used in our subsequent analysis. The main bulk of the article is contained in section 5, where the Euler-Lagrange equations associated with shell buckling are identified, and analyzed asymptotically in the limit as the shell thickness ratio tends to zero. Two distinguished limits are identified, depending on the relative order of magnitude of the two shell parameters mentioned earlier. Finally we present our results, and conclude in section 6 .

2. Problem description. A complete spherical shell of inner radius $R_{0}$ and outer radius $R_{1}$ is perfectly bonded to an elastic matrix. We will work with the shell middle-surface radius $\widehat{R}=\frac{1}{2}\left(R_{0}+R_{1}\right)$ and with the shell thickness $h=R_{1}-R_{0}$. The shell and the matrix are both treated as linearly elastic materials, with given shear moduli and Poisson ratios $G_{\mathrm{s}}, G_{\mathrm{m}}$ and $\nu_{\mathrm{s}}, \nu_{\mathrm{m}}$. Here a subscript "s" refers to the shell and " $\mathrm{m}$ " to the matrix. In the matrix we prescribe a stress field at infinity, such that the only component (in Cartesian coordinates) is $\left.\tau_{z z}\right|_{\infty}=-q$. We impose that $q>0$ so that the uniaxial stress at infinity is compressive.

We will work primarily in spherical polar coordinates, with $\theta$ denoting the polar angle and $\phi$ the azimuthal angle. The displacement that the shell undergoes under buckling is, as in [12], assumed to be axisymmetric, i.e., independent of $\phi$. The components of this buckling displacement in the normal (radial) direction and the $\theta$-direction are denoted by $w$ and $v$, respectively. The critical stress at which buckling occurs, together with the associated buckling pattern, is found by minimizing the following integral [12]:

$$
\begin{aligned}
I[w, v]=\iint_{\substack{\text { mids-shell } \\
\text { surface }}}\left\{\frac{q}{2}\left(p_{0}+p_{2} P_{2}(\cos \theta)\right)\left(\frac{\mathrm{d} w}{\mathrm{~d} \theta}\right)^{2}+\frac{h G_{\mathrm{S}}}{\widehat{R}^{2}\left(1-\nu_{\mathrm{s}}\right)}\right. \\
\times\left[\left(1-\nu_{\mathrm{s}}^{2}\right) w^{2}+\left(\frac{\mathrm{d} v}{\mathrm{~d} \theta}+v \cot \theta-\left(1+\nu_{\mathrm{s}}\right) w\right)^{2}\right. \\
\left.\left.\quad+\frac{h^{2}}{12 \widehat{R}^{2}}\left(\frac{\mathrm{d}^{2} w}{\mathrm{~d} \theta^{2}}+\cot \theta \frac{\mathrm{d} w}{\mathrm{~d} \theta}\right)^{2}\right]\right\} \mathrm{d} S \\
-\frac{1}{2} \iint_{R=R_{1}}\left(\tau_{R R}[w, v] w+\tau_{R \theta}[w, v] v\right) \mathrm{d} S
\end{aligned}
$$

Copyright $@$ by SIAM. Unauthorized reproduction of this article is prohibited. 


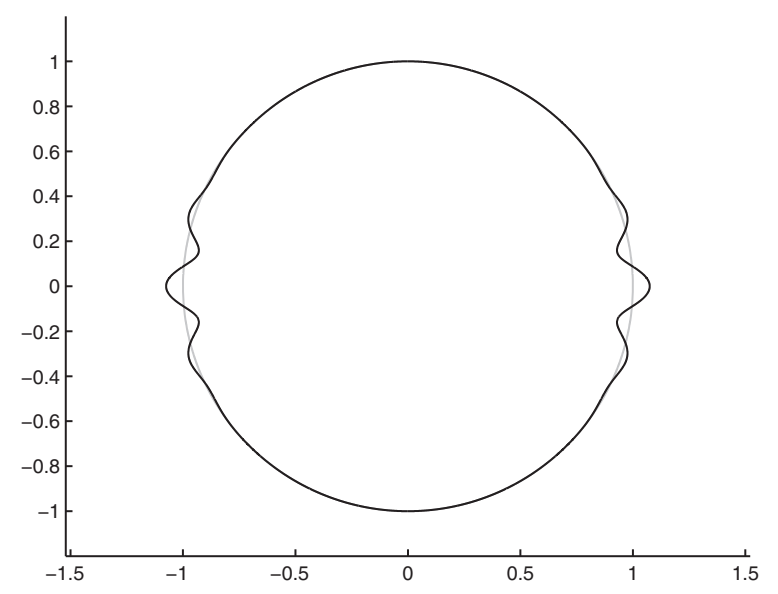

FIG. 2.1. Buckling pattern for the lowest critical stress. Image reproduced from [12] with permission from Oxford University Press.

In this expression, $P_{2}(\mu)=\left(3 \mu^{2}-1\right) / 2$ is the second-degree Legendre polynomial. The constants $p_{0}$ and $p_{2}$ are given by

$$
\begin{aligned}
& p_{0}=\frac{h G_{\mathrm{s}}}{2 \widehat{R}^{2} G_{\mathrm{m}}} \frac{\left(1-\nu_{\mathrm{m}}\right)\left(-5 \nu_{\mathrm{m}}+15 \nu_{\mathrm{m}} \nu_{\mathrm{s}}-17+3 \nu_{\mathrm{s}}\right)}{\left(1-\nu_{\mathrm{s}}\right)\left(7-5 \nu_{\mathrm{m}}\right)\left(1+\nu_{\mathrm{m}}\right)}, \\
& p_{2}=\frac{10 h G_{\mathrm{s}}\left(1-\nu_{\mathrm{m}}\right)}{\widehat{R}^{2} G_{\mathrm{m}}\left(1-\nu_{\mathrm{s}}\right)\left(7-5 \nu_{\mathrm{m}}\right)} .
\end{aligned}
$$

Finally $\tau_{R R}$ and $\tau_{R \theta}$ are two of the components of stress in the matrix, which in the integral are evaluated at the outer shell edge (which is to leading-order equivalent to the shell middle surface). These stress components depend on the shell displacements $v$ and $w$. The minimization problem relies on Koiter's shallow shell theory [13], for which the wavelength of the buckling pattern must be smaller than the radius of curvature of the undeformed shell, and larger than the shell thickness.

Some typical parameter values for the microspheres embedded in the anechoic tiles (mentioned in section 1) are that the thickness ratio $h / \widehat{R}=0.02$, the ratio of shear moduli $G_{\mathrm{s}} / G_{\mathrm{m}}=100$, and the Poisson ratios of the matrix and shell are $\nu_{\mathrm{m}}=0.45$ and $\nu_{\mathrm{s}}=0.35$, respectively. On minimizing the integral (2.1) numerically for these values by the Rayleigh-Ritz method, the lowest critical buckling stress was found to be $q=0.0550$, with the corresponding buckling pattern shown in Figure 2.1.

One numerical experiment made in [12] was to examine the limit $h / \widehat{R} \rightarrow 0$. In this limit, while keeping the other constants at their typical values described earlier, the critical buckling stress $q$ tended to a constant value while the behavior of the buckling pattern is shown in Figure 2.2. We note that as $h / \widehat{R}$ decreases, the wavelength of the buckling pattern decreases, while at the same time the extent of the buckled region also decreases. A similar pattern occurs in the case where we consider the quantity $G_{\mathrm{s}} / G_{\mathrm{m}} \rightarrow 0$, but not as rapidly, and the critical stress in this limit is unbounded. The behavior of the buckling patterns in these limits suggests a WKB approach to the eigenvalue problem, and that is the subject of this paper. 

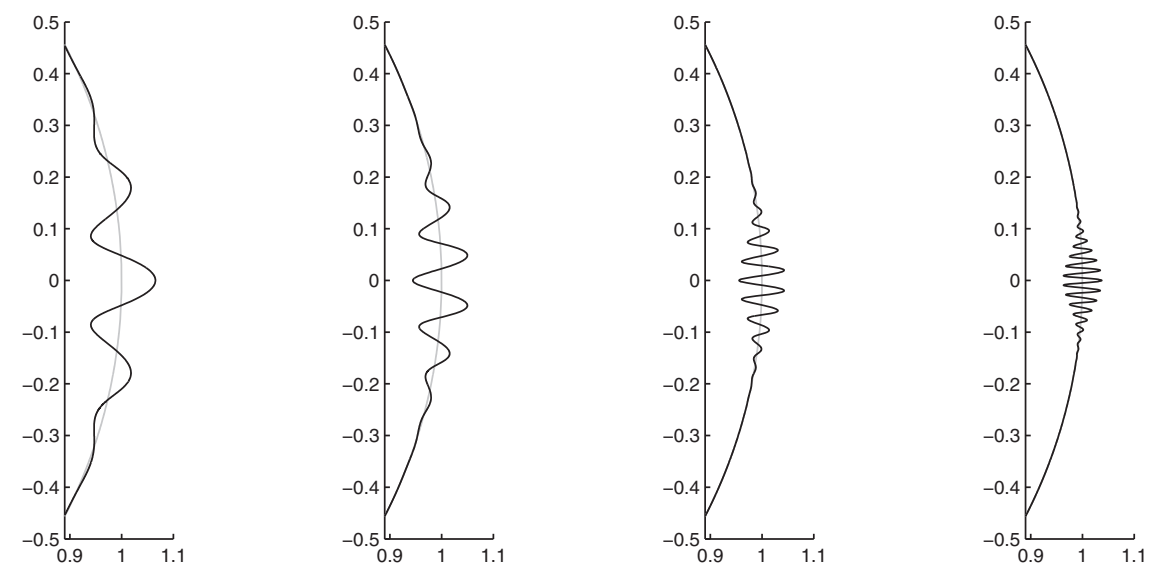

FIG. 2.2. Buckling patterns for $G_{\mathrm{s}} / G_{\mathrm{m}}=100$ and $h / \widehat{R}=0.01, \quad 0.005, \quad 0.002$, and 0.001 . Images reproduced from [12] with permission from Oxford University Press.

3. A paradigm problem. Before analyzing the buckling behavior of the shell, we consider the much simpler paradigm problem of a beam of length $2 L$ on a Winkler foundation of elastic springs, as depicted in Figure 3.1 [9]. We apply a compressive force $P$ to either end of the beam, and determine the critical value of $P$ for which the beam buckles, under clamped conditions at the ends. Problems of this type have been analyzed by Hunt et al. [11], Lee and Waas [14], and more recently by Coman [6].

If the stiffness of the substrate is $k(x)$, where $x$ is the position along the beam, then the transverse displacement $w$ of the beam is given by the solution to EI $w^{\prime \prime \prime \prime}+$ $P w^{\prime \prime}+k(x) w=0$, with boundary conditions $w( \pm L)=w^{\prime}( \pm L)=0$ [6]. We assume that $k$ is such that it has a minimum at $x=x_{0}$, or $k^{\prime}\left(x_{0}\right)=0$ and $k^{\prime \prime}\left(x_{0}\right)>0$. Without loss of generality we take $x_{0}=0$. Nondimensionalizing via $x=L \widehat{x}, k(x)=K \widehat{k}(\widehat{x})$ gives

$$
\varepsilon^{4} w^{\prime \prime \prime \prime}+2 \varepsilon^{2} \lambda w^{\prime \prime}+k(x) w=0,
$$

with $w( \pm 1)=w^{\prime}( \pm 1)=0$, where $\varepsilon^{4}=E I /\left(K L^{4}\right)$ and $\lambda=\frac{1}{2} P / \sqrt{K E I}$, and where we have dropped the $\widehat{ }$ notation for convenience.

We are interested in the solution to this eigenvalue problem in the limit as $\varepsilon \rightarrow 0$. Numerical solutions for small $\varepsilon$ show that the eigenmode associated with the lowest eigenvalue has the form of a highly oscillatory wave packet centered on the weakest part of the foundation, as shown in Figure 3.2. This pattern is similar to that seen in the limit in Figure 2.2 for the embedded shell.

3.1. Asymptotic analysis. Coman [6] solved (3.1) by substituting in a WKBtype ansatz. For problems as simple as (3.1) it is relatively straightforward to deter-

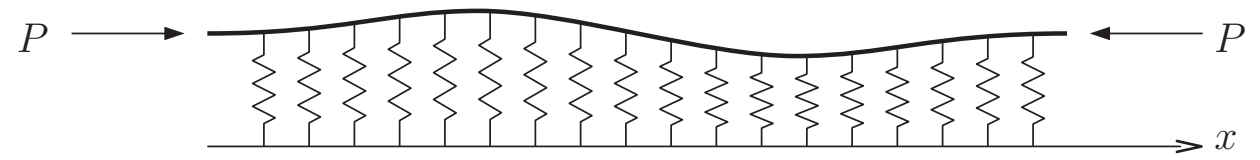

Fig. 3.1. A diagram of a beam attached to a deformable substrate, experiencing a compressive force $P$.

Copyright $@$ by SIAM. Unauthorized reproduction of this article is prohibited. 


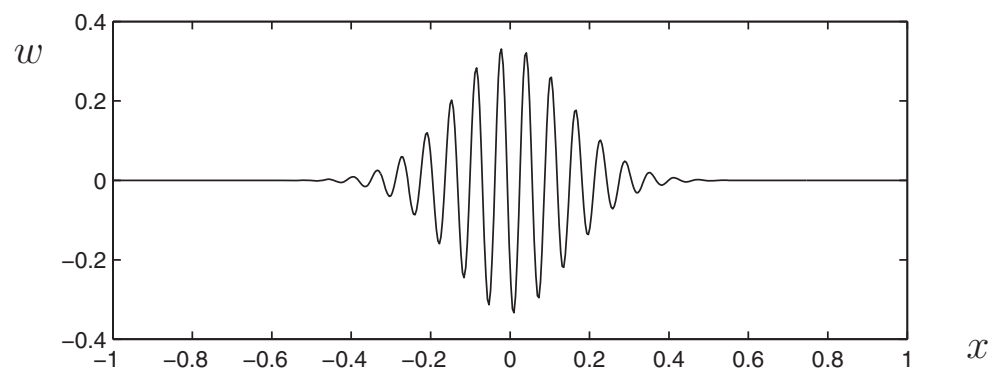

FIG. 3.2. The eigenmode associated with the lowest eigenvalue of (3.1) with clamped conditions at \pm 1 , when $\varepsilon=0.01$ and $k(x)=1+x^{2}$.

mine the scales of the fast oscillation and slow modulation, after which the standard theory of multiple scales can be used [10]. However, we will see that for the case of the embedded shell it is not so easy to determine these scales. Thus we detail briefly here the steps necessary to determine the fast and slow scales in a way which translates to the harder problem.

The first step is to identify the characteristic wavelength of the buckling pattern. Scaling $x=\varepsilon^{\alpha} \bar{x}$ we find a balance of terms when $\alpha=1$ giving

$$
w^{\prime \prime \prime \prime}+2 \lambda w^{\prime \prime}+k(0) w=0,
$$

with solutions $w=\mathrm{e}^{\mathrm{i} \kappa \bar{x}}$, where $\kappa^{2}=\lambda \pm \sqrt{\lambda^{2}-k(0)}$. At the bifurcation point $\lambda=\sqrt{k(0)}$, pairs of complex roots coalesce and become real. The existence of two real wavenumbers which are almost equal for $\lambda>\sqrt{k(0)}$ is the origin of the key lengthscale over which the oscillation is modulated (cf. beats in acoustics). Setting $\kappa=k(0)^{1 / 4}$ we now determine the scale of this slow modulation by writing $x=\varepsilon^{\gamma} \widehat{x}$, $w=\mathrm{e}^{\mathrm{i} \kappa x / \varepsilon} f(\widehat{x})$, and $\lambda=\kappa^{2}+\varepsilon^{\beta} \lambda_{1}$, to give

$$
\begin{aligned}
\varepsilon^{4-4 \gamma} f^{\prime \prime \prime \prime}+4 \mathrm{i} \varepsilon^{3-3 \gamma} \kappa f^{\prime \prime \prime}+\varepsilon^{2-2 \gamma}\left(-4 \kappa^{2}+2 \varepsilon^{\beta} \lambda_{1}\right) f^{\prime \prime}+4 \varepsilon^{1-\gamma+\beta} \mathrm{i} \kappa \lambda_{1} f^{\prime} & \\
+ & \left(-2 \varepsilon^{\beta} \kappa^{2} \lambda_{1}+\frac{\varepsilon^{2 \gamma} \widehat{x}^{2}}{2} k^{\prime \prime}(0)+O\left(\varepsilon^{3 \gamma}\right)\right) f=0,
\end{aligned}
$$

where a prime now denotes differentiation with respect to $\widehat{x}$. We see that the correct scaling is $2-2 \gamma=2 \gamma=\beta$, giving $\gamma=1 / 2, \beta=1$, and

$$
f^{\prime \prime}+\left(\frac{\lambda_{1}}{2}-\frac{k^{\prime \prime}(0) \widehat{x}^{2}}{8 \kappa^{2}}\right) f=0,
$$

with boundary conditions $f \rightarrow 0$ as $\widehat{x} \rightarrow \pm \infty$. Equation (3.4), together with this condition at infinity, is known as the quantum-mechanical harmonic oscillator problem [1] and has solution

$$
\begin{aligned}
\lambda_{1}^{(n)} & =\frac{\sqrt{k^{\prime \prime}(0)}}{\sqrt{2} k(0)^{1 / 4}}(2 n+1), \\
f_{n} & =H_{n}\left(\left[\frac{k^{\prime \prime}(0)}{8 k(0)^{1 / 2}}\right]^{1 / 4} \widehat{x}\right) \exp \left(-\frac{\sqrt{k^{\prime \prime}(0)}}{4 \sqrt{2} k(0)^{1 / 4}} \widehat{x}^{2}\right),
\end{aligned}
$$

where $H_{n}(\widetilde{x})$ is a Hermite polynomial. This corresponds exactly to Coman's results $[6]$. 
We now therefore have a general mechanism for taking a problem similar to the beam on a substrate of variable stiffness, and reducing it to a simpler eigenvalue problem. Briefly:

- We obtain the Euler-Lagrange equation.

- We identify that the buckling is centered on $x=x_{0}$, and we find the oscillation wavelength by substituting $x=x_{0}+\varepsilon^{\alpha} \bar{x}$, where $x$ is the independent variable of the equation.

- We find the leading-order equation for the wavenumber by matching leadingorder terms in the scaled Euler-Lagrange equation, thus finding $\alpha$, and by looking for an oscillatory solution $w_{0}(\bar{x})$. The leading order $\lambda$, given by $\lambda_{0}$, is found by choosing the value of $\lambda$ in this equation so that two roots coincide, and the wavenumber is set to be the value of these coincident roots.

- We substitute $w=w_{0}(\bar{x}) f(x)$ into the Euler-Lagrange equation.

- We set $\lambda=\lambda_{0}+\varepsilon^{\beta} \lambda_{1}$ and $x=x_{0}+\varepsilon^{\gamma} \widehat{x}$ for $\gamma<\alpha$. By judicious choice of $\beta$ and $\gamma$, we obtain an equation that can be scaled to give an eigenvalue problem for the amplitude $f$.

- The buckling pattern is then given by $w=w_{0}(\bar{x}) f(x)$, where $f(x)$ and the correction $\lambda_{1}$ to the critical parameter $\lambda$ are given by the solution to the eigenvalue problem for $f$.

4. WKB analysis of the Navier equations. In this section we will analyze a problem which will be used later to simplify the shell buckling equations. Recall that for the shell the boundary of the elastic matrix was curved. However, in the limit as the thickness of the shell tended to zero, the region over which buckling occurred became ever smaller, implying that we are justified in approximating the elastic matrix in the limit by a half-plane.

The geometry of the problem which we will analyze, therefore, is depicted in Figure 4.1. Given that the displacements on the boundary of the half-plane (in plane strain) are highly oscillatory functions multiplied by some slowly varying envelope, or

$$
\left.w\right|_{z=0}=\Re\left[W_{\mathrm{b}}(x) \exp \left(\frac{\mathrm{i} k x}{\varepsilon}\right)\right],\left.\quad u\right|_{z=0}=\Re\left[U_{\mathrm{b}}(x) \exp \left(\frac{\mathrm{i} k x}{\varepsilon}\right)\right],
$$

we wish to determine the stresses $\tau_{z z}$ and $\tau_{x z}$ on the boundary $z=0$. This will be achieved by first finding the displacement fields in $z>0$ and then extracting the stress components from these. The displacement fields in $z>0$ are found using the two-dimensional Navier equations,

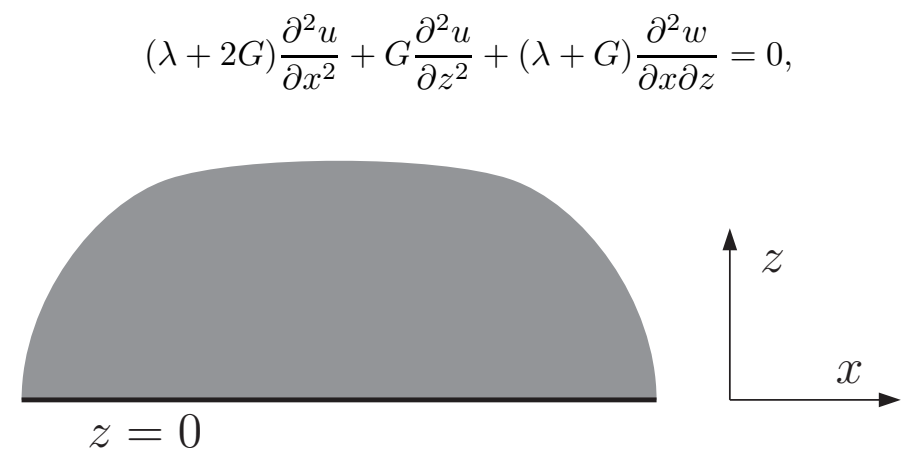

FIG. 4.1. Geometry of the plane strain problem. 


$$
G \frac{\partial^{2} w}{\partial x^{2}}+(\lambda+2 G) \frac{\partial^{2} w}{\partial z^{2}}+(\lambda+G) \frac{\partial^{2} u}{\partial x \partial z}=0 .
$$

We find it easier to calculate the displacements in terms of the Lamé constants $\lambda$ and $G$; we will return to the $(G, \nu)$ notation later.

We assume that the envelope terms on the boundary have asymptotic expansions as follows:

$$
\begin{aligned}
U_{\mathrm{b}}(x) & =U_{\mathrm{b} 0}(x)+\varepsilon U_{\mathrm{b} 1}(x)+\varepsilon^{2} U_{\mathrm{b} 2}(x)+\cdots, \\
W_{\mathrm{b}}(x) & =W_{\mathrm{b} 0}(x)+\varepsilon W_{\mathrm{b} 1}(x)+\varepsilon^{2} W_{\mathrm{b} 2}(x)+\cdots .
\end{aligned}
$$

Then, we make an ansatz

$$
u=\Re\left[U(x, z) \exp \left(\frac{\phi(x, z)}{\varepsilon}\right)\right], \quad w=\Re\left[W(x, z) \exp \left(\frac{\phi(x, z)}{\varepsilon}\right)\right],
$$

where again the envelopes have asymptotic expansions of the form

$$
\begin{aligned}
U(x, z) & =\frac{1}{\varepsilon} U_{-1}(x, z)+U_{0}(x, z)+\varepsilon U_{1}(x, z)+\cdots, \\
W(x, z) & =\frac{1}{\varepsilon} W_{-1}(x, z)+W_{0}(x, z)+\varepsilon W_{1}(x, z)+\cdots .
\end{aligned}
$$

The scaling of the first term with $\varepsilon^{-1}$ is made with the benefit of hindsight; this is necessary so that terms can be matched to the boundary conditions. $O\left(\varepsilon^{-3}\right)$,

Substituting the ansatz (4.6) into the Navier equations (4.2)-(4.3) we find at

$$
\begin{aligned}
(\lambda+2 G) \phi_{x}^{2} U_{-1}+G \phi_{z}^{2} U_{-1}+(\lambda+G) \phi_{x} \phi_{z} W_{-1} & =0 \\
G \phi_{x}^{2} W_{-1}+(\lambda+2 G) \phi_{z}^{2} W_{-1}+(\lambda+G) \phi_{x} \phi_{z} U_{-1} & =0 .
\end{aligned}
$$

A nontrivial solution exists providing $G(\lambda+2 G)\left(\phi_{x}^{2}+\phi_{z}^{2}\right)^{2}=0$, i.e., $\phi_{x}+\sigma \mathrm{i} \phi_{z}=0$, where $\sigma= \pm 1$. Thus the rays associated with the WKB ansatz (4.6) are complex [4]. The boundary conditions $\left.\phi\right|_{z=0}=\mathrm{i} k x, \Re(\phi) \rightarrow-\infty \quad$ as $z \rightarrow+\infty$ imply

$$
\phi(x, z)=\mathrm{i} k x-\sigma k z,
$$

where $\sigma=\operatorname{sign}(k)$. Then (4.9)-(4.10) give $U_{-1}+\mathrm{i} \sigma W_{-1}=0$.

Now we look at $O\left(\varepsilon^{n-2}\right)$ in the Navier equations. Using the expression (4.11) for $\phi(x, z)$, the two equations become

$$
\begin{aligned}
(\lambda+G) k^{2}\left(U_{n}+\mathrm{i} \sigma W_{n}\right)= & (\lambda+2 G) U_{(n-2), x x}+G U_{(n-2), z z}+(\lambda+G) W_{(n-2), x z} \\
& +2 \mathrm{i} k(\lambda+2 G) U_{(n-1), x}-2 \sigma k G U_{(n-1), z} \\
& -\sigma k(\lambda+G) W_{(n-1), x}+\mathrm{i} k(\lambda+G) W_{(n-1), z}
\end{aligned}
$$

$(\lambda+G) k^{2}\left(U_{n}+\mathrm{i} \sigma W_{n}\right)=-\mathrm{i} \sigma(\lambda+G) U_{(n-2), x z}-\mathrm{i} \sigma G W_{(n-2), x x}-\mathrm{i} \sigma(\lambda+2 G) W_{(n-2), z z}$

$$
\begin{aligned}
& +\mathrm{i} k(\lambda+G) U_{(n-1), x}+\sigma k(\lambda+G) U_{(n-1), z} \\
& +2 \sigma k G W_{(n-1), x}+2 \mathrm{i} k(\lambda+2 G) W_{(n-1), z},
\end{aligned}
$$

where a subscript preceded with a comma indicates partial differentiation and by convention $U_{-2}=W_{-2}=U_{-3}=W_{-3}=\cdots=0$. 
At this stage, it is convenient to introduce the new independent variables $\xi=$ $\mathrm{i} \sigma z-x, \eta=\mathrm{i} \sigma z+x$, and the new dependent variables $a_{n}=U_{n}+\mathrm{i} \sigma W_{n}, b_{n}=U_{n}-\mathrm{i} \sigma W_{n}$. Then (4.12)-(4.13) become

$$
\begin{aligned}
(\lambda+G) k^{2} a_{n}= & (\lambda+G) a_{n-2, \eta \eta}-(\lambda+3 G) a_{n-2, \xi \eta}-(\lambda+3 G) b_{n-2, \xi \eta}+(\lambda+G) b_{n-2, \xi \xi} \\
& +2 \mathrm{i} k(\lambda+G) a_{n-1, \eta}-\mathrm{i} k(\lambda+3 G) a_{n-1, \xi}-\mathrm{i} k(\lambda+3 G) b_{n-1, \xi}, \\
(\lambda .14) & (\lambda+G) a_{n-2, \eta \eta}+(\lambda+3 G) a_{n-2, \xi \eta}-(\lambda+3 G) b_{n-2, \xi \eta}-(\lambda+G) b_{n-2, \xi \xi} \\
(\lambda+G) k^{2} a_{n}= & +2 \mathrm{i} k(\lambda+G) a_{n-1, \eta}+\mathrm{i} k(\lambda+3 G) a_{n-1, \xi}-\mathrm{i} k(\lambda+3 G) b_{n-1, \xi} .
\end{aligned}
$$

Either of these equations can be used to determine $a_{n}$ in terms of $a_{n-1}, b_{n-1}, a_{n-2}$, and $b_{n-2}$. On the other hand $b_{n}$ is determined by the solvability condition that the right-hand sides of (4.14)-(4.15) are equal. Subtracting (4.14) from (4.15) we find

$$
(\lambda+3 G) a_{n-2, \xi \eta}-(\lambda+G) b_{n-2, \xi \xi}+\mathrm{i} k(\lambda+3 G) a_{n-1, \xi}=0 .
$$

Integrating and reindexing gives

$$
\mathrm{i} k(\lambda+3 G) a_{n}=-(\lambda+3 G) a_{n-1, \eta}+(\lambda+G) b_{n-1, \xi}+f_{n}(\eta),
$$

where $f_{n}$ is an arbitrary function of $\eta$.

Now eliminating $a_{n}$ between (4.17) and (4.14) and reindexing gives

$$
\begin{gathered}
(\lambda+G)\left(a_{n-1, \eta \eta}+b_{n-1, \xi \xi}\right)-(\lambda+3 G)\left(a_{n-1, \xi \eta}+b_{n-1, \xi \eta}\right)=\mathrm{i} k(\lambda+3 G) a_{n, \xi} \\
-\mathrm{i} k(\lambda+G) a_{n, \eta}+\mathrm{i} k\left[(\lambda+3 G)-\frac{(\lambda+G)^{2}}{(\lambda+3 G)}\right] b_{n, \xi}-\frac{\mathrm{i} k(\lambda+G)}{(\lambda+3 G)} f_{n+1}(\eta) .
\end{gathered}
$$

Using this equation allows us to determine $b_{n}$ in terms of $a_{n-1}, b_{n-1}, a_{n-2}, b_{n-2}$, and $a_{n}$.

By induction, one can show that this process yields the solution

$$
\begin{aligned}
& a_{n}(\xi, \eta)=-\frac{\lambda+3 G}{\lambda+G} \sum_{m=1}^{n+1}\left(\frac{\mathrm{i}}{k}\right)^{m} \mathscr{F}_{n-m}^{(m-1)}(\eta), \\
& b_{n}(\xi, \eta)=\xi \mathscr{F}_{n}(\eta)+\mathscr{G}_{n}(\eta),
\end{aligned}
$$

where $\mathscr{F}_{n}(\eta)$ and $\mathscr{G}_{n}(\eta)$ are arbitrary functions for each $n$ (with $\mathscr{F}_{n}$ proportional to $\left.f_{n+1}\right)$. We solve for these functions by applying the boundary conditions on $z=0$, giving us

$$
\begin{aligned}
\mathscr{F}_{n}(\eta) & =\frac{\lambda+G}{\lambda+3 G}\left[U_{\mathrm{b} n}^{\prime}(\eta)+\mathrm{i} \sigma W_{\mathrm{b} n}^{\prime}(\eta)+\mathrm{i} k\left(U_{\mathrm{b}(n+1)}(\eta)+\mathrm{i} \sigma W_{\mathrm{b}(n+1)}(\eta)\right)\right], \\
\mathscr{G}_{n}(\eta) & =U_{\mathrm{b} n}(\eta)-\mathrm{i} \sigma W_{\mathrm{b} n}(\eta)+\eta \mathscr{F}_{n}(\eta) .
\end{aligned}
$$

Collecting all these results and changing variables back to $(x, z)$ gives us

$$
\begin{aligned}
U_{n}(x, z) & =U_{\mathrm{b} n}(x+\mathrm{i} \sigma z)+\mathrm{i} \sigma z \mathscr{F}_{n}(x+\mathrm{i} \sigma z), \\
W_{n}(x, z) & =W_{\mathrm{b} n}(x+\mathrm{i} \sigma z)-z \mathscr{F}_{n}(x+\mathrm{i} \sigma z) .
\end{aligned}
$$

These terms can now be substituted back into (4.6) to obtain the full asymptotic expression for the displacements in the half-plane. This we can then use to obtain the 
stresses in the material, and in particular the tangential and normal stresses at the boundary. Here we merely note the results, namely that

$$
\left.\tau_{z z}\right|_{z=0}=\Re \sum_{n=-1}^{\infty} \varepsilon^{n} P_{n}(x) \mathrm{e}^{\mathrm{i} k x / \varepsilon},\left.\quad \tau_{x z}\right|_{z=0}=\Re \sum_{n=-1}^{\infty} \varepsilon^{n} Q_{n}(x) \mathrm{e}^{\mathrm{i} k x / \varepsilon},
$$

where

$$
\begin{aligned}
P_{n}(x)=- & \frac{2 G^{2}}{\lambda+3 G}\left(U_{\mathrm{b} n}^{\prime}(x)+\mathrm{i} k U_{\mathrm{b}(n+1)}(x)\right) \\
+ & \mathrm{i} \sigma \frac{2 G(\lambda+2 G)}{\lambda+3 G}\left(W_{\mathrm{b} n}^{\prime}(x)+\mathrm{i} k W_{\mathrm{b}(n+1)}(x)\right), \\
Q_{n}(x)= & \mathrm{i} \sigma \frac{2 G(\lambda+2 G)}{\lambda+3 G}\left(U_{\mathrm{b} n}^{\prime}(x)+\mathrm{i} k U_{\mathrm{b}(n+1)}(x)\right) \\
& +\frac{2 G^{2}}{\lambda+3 G}\left(W_{\mathrm{b} n}^{\prime}(x)+\mathrm{i} k W_{\mathrm{b}(n+1)}(x)\right) .
\end{aligned}
$$

Writing the coefficients in terms of $G$ and $\nu$, and substituting (4.26)-(4.27) into (4.25), it can be shown that if the displacement on the boundary is given by an asymptotic expansion of the form (4.1), then the following relations hold to all orders,

$$
\begin{aligned}
& \left.\tau_{z z}^{\mathrm{c}}\right|_{z=0}=-\frac{2 G(1-2 \nu)}{(3-4 \nu)} \frac{\mathrm{d}}{\mathrm{d} x}\left(\left.u^{\mathrm{c}}\right|_{z=0}\right)+\mathrm{i} \sigma \frac{4 G(1-\nu)}{(3-4 \nu)} \frac{\mathrm{d}}{\mathrm{d} x}\left(\left.w^{\mathrm{c}}\right|_{z=0}\right), \\
& \left.\tau_{x z}^{\mathrm{c}}\right|_{z=0}=\mathrm{i} \sigma \frac{4 G(1-\nu)}{(3-4 \nu)} \frac{\mathrm{d}}{\mathrm{d} x}\left(\left.u^{\mathrm{c}}\right|_{z=0}\right)+\frac{2 G(1-2 \nu)}{(3-4 \nu)} \frac{\mathrm{d}}{\mathrm{d} x}\left(\left.w^{\mathrm{c}}\right|_{z=0}\right),
\end{aligned}
$$

where $\boldsymbol{\tau}=\Re \boldsymbol{\tau}^{\mathrm{c}}$ and $\boldsymbol{u}=\Re \boldsymbol{u}^{\mathrm{c}}$.

5. The Euler-Lagrange equations for shell buckling. We will now find the Euler-Lagrange equations associated with the minimization of (2.1). In the usual manner, we will form the variational derivative

$$
\mathrm{D} I=\lim _{\delta \rightarrow 0} \frac{I[w+\delta \widetilde{w}, v+\delta \widetilde{v}]-I[w, v]}{\delta},
$$

where $\widetilde{w}, \widetilde{v}$ are trial displacements. The quantity $\mathrm{D} I$ is then set to zero for all possible trial displacements, yielding (in this case) two Euler-Lagrange equations.

We denote the integrals in $(2.1)$ by $I_{1}, I_{2}$, respectively, and consider $\mathrm{D} I_{1}, \mathrm{D} I_{2}$ in turn. Beginning with $I_{1}$, we first change variables to $\mu=\cos \theta$ and perform the $\phi$-integration to obtain

$$
\begin{aligned}
I_{1}[w, v]=2 \pi \widehat{R}^{2} \int_{-1}^{1}\{ & \frac{q}{2}\left(p_{0}+p_{2} P_{2}(\mu)\right)\left(1-\mu^{2}\right)\left(\frac{\mathrm{d} w}{\mathrm{~d} \mu}\right)^{2}+\frac{h G_{\mathrm{S}}}{\widehat{R}^{2}\left(1-\nu_{\mathrm{s}}\right)} \\
\times\left[\left(1-\nu_{\mathrm{s}}^{2}\right) w^{2}+\right. & \left(\sqrt{1-\mu^{2}} \frac{\mathrm{d} v}{\mathrm{~d} \mu}-\frac{\mu v}{\sqrt{1-\mu^{2}}}+\left(1+\nu_{\mathrm{s}}\right) w\right)^{2} \\
& \left.\left.+\frac{h^{2}}{12 \widehat{R}^{2}}\left(\left(1-\mu^{2}\right) \frac{\mathrm{d}^{2} w}{\mathrm{~d} \mu^{2}}-2 \mu \frac{\mathrm{d} w}{\mathrm{~d} \mu}\right)^{2}\right]\right\} \mathrm{d} \mu .
\end{aligned}
$$

Copyright $@$ by SIAM. Unauthorized reproduction of this article is prohibited. 
Using this expression, we find that

$$
\begin{aligned}
& \mathrm{D} I_{1}=2 \pi \widehat{R}^{2} \int_{-1}^{1}\{-q \widetilde{w} \frac{\mathrm{d}}{\mathrm{d} \mu}\left[\left(p_{0}+p_{2} P_{2}(\mu)\right)\left(1-\mu^{2}\right) \frac{\mathrm{d} w}{\mathrm{~d} \mu}\right]+\frac{2 h G_{\mathrm{S}}}{\widehat{R}^{2}\left(1-\nu_{\mathrm{s}}\right)} \\
& \times\left[\widetilde{v}\left(-\left(1-\mu^{2}\right) \frac{\mathrm{d}^{2} v}{\mathrm{~d} \mu^{2}}+2 \mu \frac{\mathrm{d} v}{\mathrm{~d} \mu}+\frac{v}{1-\mu^{2}}-\left(1+\nu_{\mathrm{s}}\right) \sqrt{1-\mu^{2}} \frac{\mathrm{d} w}{\mathrm{~d} \mu}\right)\right. \\
&+\widetilde{w}\left(\left(1+\nu_{\mathrm{s}}\right)\left(\sqrt{1-\mu^{2}} \frac{\mathrm{d} v}{\mathrm{~d} \mu}-\frac{\mu v}{\sqrt{1-\mu^{2}}}+2 w\right)\right. \\
&\left.\left.\left.\quad+\frac{h^{2}}{12 \widehat{R}^{2}} \frac{\mathrm{d}}{\mathrm{d} \mu}\left[\left(1-\mu^{2}\right) \frac{\mathrm{d}^{2}}{\mathrm{~d} \mu^{2}}\left(\left(1-\mu^{2}\right) \frac{\mathrm{d} w}{\mathrm{~d} \mu}\right)\right]\right)\right]\right\} \mathrm{d} \mu .
\end{aligned}
$$

$\mathrm{D} I_{2}=-\frac{1}{2} \lim _{\delta \rightarrow 0} \iint_{R=R_{1}}\left(\tau_{R R}[w, v] \widetilde{w}+\tau_{R \theta}[w, v] \widetilde{v}+\tau_{R R}[\widetilde{w}, \widetilde{v}] w+\tau_{R \theta}[\widetilde{w}, \widetilde{v}] v+O(\delta)\right) \mathrm{d} S$.

Now, the reciprocity theorem in linear elasticity states that if $\boldsymbol{u}_{1}$ and $\boldsymbol{u}_{2}$ are two admissable displacement fields for a given body $\Omega$, with corresponding strain fields $\boldsymbol{e}_{i}$ and stress fields $\boldsymbol{\tau}_{i}$, then we have that $\iiint_{\Omega} \boldsymbol{\tau}_{1}: \boldsymbol{e}_{2} \mathrm{~d} V=\iiint_{\Omega} \boldsymbol{\tau}_{2}: \boldsymbol{e}_{1} \mathrm{~d} V$, where $\boldsymbol{A}: \boldsymbol{B}=\sum_{i, j} A_{i j} B_{i j}$ is the scalar tensor product. Equivalently, the theorem may be applied on the surface $\Sigma=\partial \Omega$ with normal $\boldsymbol{n}$, or $\iint_{\Sigma} \boldsymbol{u}_{1} \cdot\left(\boldsymbol{\tau}_{2} \boldsymbol{n}\right) \mathrm{d} S=\iint_{\Sigma} \boldsymbol{u}_{2} \cdot\left(\boldsymbol{\tau}_{1} \boldsymbol{n}\right) \mathrm{d} S$. In our case the region $\Omega$ in which the displacements are defined is the exterior of a sphere with radius $R_{1}$. The reciprocity theorem has been proven in such exterior regions by Gurtin and Sternberg [8], assuming appropriate decay conditions on the displacement and stress components at infinity. Applying the surface reciprocity result to (5.4), where one displacement field is $(w, v)$ and the other $(\widetilde{w}, \widetilde{v})$, we have

$$
\mathrm{D} I_{2}=-2 \pi \widehat{R}^{2} \int_{-1}^{1}\left(\widetilde{w} \tau_{R R}[w, v]+\widetilde{v} \tau_{R \theta}[w, v]\right) \mathrm{d} \mu,
$$

assuming that the displacements on $R=R_{1}$ may be approximated by displacements on $R=\widehat{R}$, the center surface of the shell.

Combining (5.3) and (5.5) and equating the resulting integral $\mathrm{D} I=\mathrm{D} I_{1}+\mathrm{D} I_{2}$ to zero for every possible trial displacement $(\widetilde{w}, \widetilde{v})$, we obtain two Euler-Lagrange equations:

$$
\begin{gathered}
\frac{h^{3} G_{\mathrm{s}}}{6 \widehat{R}^{4}\left(1-\nu_{\mathrm{s}}\right)} \frac{\mathrm{d}}{\mathrm{d} \mu}\left[\left(1-\mu^{2}\right) \frac{\mathrm{d}^{2}}{\mathrm{~d} \mu^{2}}\left(\left(1-\mu^{2}\right) \frac{\mathrm{d} w}{\mathrm{~d} \mu}\right)\right]+\frac{2 h G_{\mathrm{s}}\left(1+\nu_{\mathrm{s}}\right)}{\widehat{R}^{2}\left(1-\nu_{\mathrm{s}}\right)}\left(\sqrt{1-\mu^{2}} \frac{\mathrm{d} v}{\mathrm{~d} \mu}\right. \\
\left.-\frac{\mu v}{\sqrt{1-\mu^{2}}}+2 w\right)-q \frac{\mathrm{d}}{\mathrm{d} \mu}\left[\left(p_{0}+p_{2} P_{2}(\mu)\right)\left(1-\mu^{2}\right) \frac{\mathrm{d} w}{\mathrm{~d} \mu}\right]-\tau_{R R}[w, v]=0
\end{gathered}
$$

$$
\frac{-2 h G_{\mathrm{s}}}{\widehat{R}^{2}\left(1-\nu_{\mathrm{s}}\right)}\left[\frac{\mathrm{d}}{\mathrm{d} \mu}\left(\left(1-\mu^{2}\right) \frac{\mathrm{d} v}{\mathrm{~d} \mu}\right)-\frac{v}{1-\mu^{2}}+\left(1+\nu_{\mathrm{s}}\right) \sqrt{1-\mu^{2}} \frac{\mathrm{d} w}{\mathrm{~d} \mu}\right]-\tau_{R \theta}[w, v]=0 .
$$

Next we use the theory of section 4 to write the stress terms $\tau_{R R}$ and $\tau_{R \theta}$ in terms of $w$ and $v$. Recall that if the displacement on the boundary of a half-plane 
is an amplitude-modulated highly oscillatory function, with given wavenumber $k / \varepsilon$, then the stresses on the boundary are given by (4.28)-(4.29). Our rationale for using this result is that as the thickness ratio tends to zero, numerical calculations show that the region over which buckling occurs becomes ever smaller. Thus, if we look at the scale of the disturbance in this limit, the relative curvature of the shell becomes smaller, so that in the limit the curved boundary of the matrix can be considered to be a plane. Axisymmetry in the problem ensures that two dimensions are sufficient to describe the deformation of this plane. We will assume that the condition that the boundary displacements must be amplitude-modulated highly oscillatory functions is satisfied. Thus, in order to apply (4.28)-(4.29) to the shell problem, we identify the boundary displacements $\left.w\right|_{z=0},\left.u\right|_{z=0}$ with the respective shell displacements $w, v$; the coordinate $x$ along the half-plane corresponds to the shell arc-length $s=\widehat{R} \theta$, and the stress components $\tau_{z z}, \tau_{x z}$ on the boundary are replaced by the stress components on the matrix boundary, $\tau_{R R}$ and $\tau_{R \theta}$, respectively. Recalling $\boldsymbol{\tau}=\Re \boldsymbol{\tau}^{\mathrm{c}}$ and $\boldsymbol{u}=\Re \boldsymbol{u}^{\mathrm{c}}$, these are then given by

$$
\begin{gathered}
\left.\tau_{R R}^{\mathrm{c}}\right|_{R=R_{1}}=-\frac{2 G_{\mathrm{m}}\left(1-2 \nu_{\mathrm{m}}\right)}{\widehat{R}\left(3-4 \nu_{\mathrm{m}}\right)} \frac{\mathrm{d} v^{\mathrm{c}}}{\mathrm{d} \theta}+\mathrm{i} \sigma \frac{4 G_{\mathrm{m}}\left(1-\nu_{\mathrm{m}}\right)}{\widehat{R}\left(3-4 \nu_{\mathrm{m}}\right)} \frac{\mathrm{d} w^{\mathrm{c}}}{\mathrm{d} \theta}, \\
\left.\tau_{R \theta}^{\mathrm{c}}\right|_{R=R_{1}}=\mathrm{i} \sigma \frac{4 G_{\mathrm{m}}\left(1-\nu_{\mathrm{m}}\right)}{\widehat{R}\left(3-4 \nu_{\mathrm{m}}\right)} \frac{\mathrm{d} v^{\mathrm{c}}}{\mathrm{d} \theta}+\frac{2 G_{\mathrm{m}}\left(1-2 \nu_{\mathrm{m}}\right)}{\widehat{R}\left(3-4 \nu_{\mathrm{m}}\right)} \frac{\mathrm{d} w^{\mathrm{c}}}{\mathrm{d} \theta} .
\end{gathered}
$$

We now drop the.$^{c}$ notation, and assume henceforth that the displacements are given by the real parts of $w, v$. The expressions (5.8)-(5.9) are substituted into (5.6)-(5.7), together with the transformation $\mu=\cos \theta$, to give

$$
\begin{gathered}
\frac{h^{3} G_{\mathrm{s}}}{6 \widehat{R}^{4}\left(1-\nu_{\mathrm{s}}\right)} \frac{\mathrm{d}}{\mathrm{d} \mu}\left[\left(1-\mu^{2}\right) \frac{\mathrm{d}^{2}}{\mathrm{~d} \mu^{2}}\left(\left(1-\mu^{2}\right) \frac{\mathrm{d} w}{\mathrm{~d} \mu}\right)\right]-q \frac{\mathrm{d}}{\mathrm{d} \mu}\left[\left(p_{0}+p_{2} P_{2}(\mu)\right)\left(1-\mu^{2}\right) \frac{\mathrm{d} w}{\mathrm{~d} \mu}\right] \\
+\frac{2 h G_{\mathrm{s}}\left(1+\nu_{\mathrm{s}}\right)}{\widehat{R}^{2}\left(1-\nu_{\mathrm{s}}\right)}\left[\frac{\mathrm{d}}{\mathrm{d} \mu}\left(\sqrt{1-\mu^{2}} v\right)+2 w\right]-\frac{2 G_{\mathrm{m}}\left(1-2 \nu_{\mathrm{m}}\right)}{\widehat{R}\left(3-4 \nu_{\mathrm{m}}\right)} \sqrt{1-\mu^{2}} \frac{\mathrm{d} v}{\mathrm{~d} \mu} \\
+\mathrm{i} \sigma \frac{4 G_{\mathrm{m}}\left(1-\nu_{\mathrm{m}}\right)}{\widehat{R}\left(3-4 \nu_{\mathrm{m}}\right)} \sqrt{1-\mu^{2}} \frac{\mathrm{d} w}{\mathrm{~d} \mu}=0, \\
\frac{2 h G_{\mathrm{s}}}{\widehat{R}^{2}\left(1-\nu_{\mathrm{s}}\right)}\left[-\frac{\mathrm{d}}{\mathrm{d} \mu}\left(\left(1-\mu^{2}\right) \frac{\mathrm{d} v}{\mathrm{~d} \mu}\right)+\frac{v}{1-\mu^{2}}-\left(1+\nu_{\mathrm{s}}\right) \sqrt{1-\mu^{2}} \frac{\mathrm{d} w}{\mathrm{~d} \mu}\right] \\
+\mathrm{i} \sigma \frac{4 G_{\mathrm{m}}\left(1-\nu_{\mathrm{m}}\right)}{\widehat{R}\left(3-4 \nu_{\mathrm{m}}\right)} \sqrt{1-\mu^{2}} \frac{\mathrm{d} v}{\mathrm{~d} \mu}+\frac{2 G_{\mathrm{m}}\left(1-2 \nu_{\mathrm{m}}\right)}{\widehat{R}\left(3-4 \nu_{\mathrm{m}}\right)} \sqrt{1-\mu^{2}} \frac{\mathrm{d} w}{\mathrm{~d} \mu}=0 .
\end{gathered}
$$

5.1. Nondimensionalization. We now take the equations above and nondimensionalize them. With dimensionless quantities signified by a prime, we set $w=$ $W w^{\prime}, v=\eta W v^{\prime}, q=G_{\mathrm{m}} Q q^{\prime}$, and $p_{i}=\frac{h G_{\mathrm{s}}}{\widehat{R}^{2} G_{\mathrm{m}}} p_{i}^{\prime}$, where $\eta$ and $Q$ are dimensionless scalings to be chosen and $W$ is an arbitrary length scale. On further introducing the thickness ratio $\varepsilon=h / \widehat{R}$ and the shear modulus ratio $\mathscr{G}=G_{\mathrm{s}} / G_{\mathrm{m}}$, the Euler-Lagrange equations adopt their nondimensional form,

$$
\begin{aligned}
& \varepsilon^{3} \mathscr{G} \frac{\mathrm{d}}{\mathrm{d} \mu}\left[\left(1-\mu^{2}\right) \frac{\mathrm{d}^{2}}{\mathrm{~d} \mu^{2}}\left(\left(1-\mu^{2}\right) \frac{\mathrm{d} w}{\mathrm{~d} \mu}\right)\right]+\varepsilon \mathscr{G} Q \cdot q \frac{\mathrm{d}}{\mathrm{d} \mu}\left[\left(A-B \mu^{2}\right)\left(1-\mu^{2}\right) \frac{\mathrm{d} w}{\mathrm{~d} \mu}\right] \\
+ & 12 \varepsilon \mathscr{G}\left(1+\nu_{\mathrm{s}}\right)\left[\eta \frac{\mathrm{d}}{\mathrm{d} \mu}\left(\sqrt{1-\mu^{2}} v\right)+2 w\right]+\mathrm{i} \sigma C \sqrt{1-\mu^{2}} \frac{\mathrm{d} w}{\mathrm{~d} \mu}-\eta D \sqrt{1-\mu^{2}} \frac{\mathrm{d} v}{\mathrm{~d} \mu}=0,
\end{aligned}
$$




$$
\begin{aligned}
12 \varepsilon \mathscr{G}\left[-\eta \frac{\mathrm{d}}{\mathrm{d} \mu}\left(\left(1-\mu^{2}\right) \frac{\mathrm{d} v}{\mathrm{~d} \mu}\right)+\right. & \left.\frac{\eta v}{1-\mu^{2}}-\left(1+\nu_{\mathrm{s}}\right) \sqrt{1-\mu^{2}} \frac{\mathrm{d} w}{\mathrm{~d} \mu}\right] \\
& +D \sqrt{1-\mu^{2}} \frac{\mathrm{d} w}{\mathrm{~d} \mu}+\mathrm{i} \sigma \eta C \sqrt{1-\mu^{2}} \frac{\mathrm{d} v}{\mathrm{~d} \mu}=0,
\end{aligned}
$$

where the prime notation has been dropped for convenience. Note that we have introduced the notation $A-B \mu^{2}=-6\left(1-\nu_{\mathrm{s}}\right)\left(p_{0}^{\prime}+p_{2}^{\prime} P_{2}(\mu)\right)$, so that the positive quantities $A$ and $B$ are given by

$$
A=\frac{9\left(1-\nu_{\mathrm{m}}\right)\left(9+5 \nu_{\mathrm{m}}-5 \nu_{\mathrm{m}} \nu_{\mathrm{s}}-\nu_{\mathrm{s}}\right)}{\left(7-5 \nu_{\mathrm{m}}\right)\left(1+\nu_{\mathrm{m}}\right)}, \quad B=\frac{90\left(1-\nu_{\mathrm{m}}\right)}{7-5 \nu_{\mathrm{m}}} .
$$

Furthermore, the positive constants $C$ and $D$ are given by

$$
C=\frac{24\left(1-\nu_{\mathrm{s}}\right)\left(1-\nu_{\mathrm{m}}\right)}{3-4 \nu_{\mathrm{m}}}, \quad D=\frac{12\left(1-\nu_{\mathrm{s}}\right)\left(1-2 \nu_{\mathrm{m}}\right)}{3-4 \nu_{\mathrm{m}}} .
$$

5.2. Identifying the distinguished limits. In this section we apply the first step in the method identified at the end of section 3. In other words, we take the dimensionless Euler-Lagrange equations (5.12)-(5.13) and set $\mu=\mu_{0}+\varepsilon^{\alpha} \bar{\mu}$, in order to obtain the wavenumber of the oscillatory component of the buckling pattern. Clearly this pattern is centered at the equator $(\mu=0)$, and so we set $\mu_{0}=0$. Retaining only the largest terms, the equations become

$$
\begin{aligned}
& \varepsilon^{3-4 \alpha} \mathscr{G} \frac{\mathrm{d}^{4} w}{\mathrm{~d} \bar{\mu}^{4}}+12 \varepsilon^{1-\alpha} \mathscr{G}\left(1+\nu_{\mathrm{s}}\right)\left(\eta \frac{\mathrm{d} v}{\mathrm{~d} \bar{\mu}}+2 \varepsilon^{\alpha} w\right) \\
& +\varepsilon^{1-2 \alpha} \mathscr{G} Q \cdot A q \frac{\mathrm{d}^{2} w}{\mathrm{~d} \bar{\mu}^{2}}+\varepsilon^{-\alpha} \mathrm{i} \sigma C \frac{\mathrm{d} w}{\mathrm{~d} \bar{\mu}}-\eta \varepsilon^{-\alpha} D \frac{\mathrm{d} v}{\mathrm{~d} \bar{\mu}}=0 \\
& -12 \mathscr{G} \eta \varepsilon^{1-2 \alpha} \frac{\mathrm{d}^{2} v}{\mathrm{~d} \bar{\mu}^{2}}-12 \mathscr{G} \varepsilon^{1-\alpha}\left(1+\nu_{\mathrm{s}}\right) \frac{\mathrm{d} w}{\mathrm{~d} \bar{\mu}}+\eta \varepsilon^{-\alpha} \mathrm{i} \sigma C \frac{\mathrm{d} v}{\mathrm{~d} \bar{\mu}}+\varepsilon^{-\alpha} D \frac{\mathrm{d} w}{\mathrm{~d} \bar{\mu}}=0
\end{aligned}
$$

We now need to choose the scaling parameters $Q$ and $\eta$, to determine $\varepsilon$ in terms of $\mathscr{G}$, and to determine $\alpha$. In the first equation, we choose $Q$ to balance the terms $w^{\prime \prime \prime \prime}$, $A q w^{\prime \prime}$, and $\mathscr{G}$ to balance these with the term i $\sigma C w^{\prime}$. This gives us $O\left(\varepsilon^{3-4 \alpha C \mathscr{G}}\right)=$ $O\left(\varepsilon^{1-2 \alpha} \mathscr{G} Q\right)=O\left(\varepsilon^{-\alpha}\right)$ as $\varepsilon \rightarrow 0$, giving $\mathscr{G}=O\left(\varepsilon^{3 \alpha-3}\right), Q=O\left(\varepsilon^{2-2 \alpha}\right)$. Since $Q$ is a scaling parameter we are free to set $Q=\varepsilon^{2-2 \alpha}$. However, $\mathscr{G}$ is the ratio of shear moduli and thus cannot be prescribed. We can, however, set

$$
\mathscr{G}=\varepsilon^{3 \alpha-3} \Gamma
$$

where $\Gamma=O(1)$. Thus, given a thickness ratio $\varepsilon$ and a shear modulus ratio $\mathscr{G}$, the scaling $\alpha$ should be chosen so as to make $\Gamma$ an $O(1)$ parameter in the expression (5.18).

Substituting for $\mathscr{G}$ and $Q$ in (5.16)-(5.17) and rearranging, we find that

$$
\begin{array}{r}
w^{\prime \prime \prime \prime}+12 \eta \varepsilon^{3 \alpha-2}\left(1+\nu_{\mathrm{s}}\right) v^{\prime}+24 \varepsilon^{4 \alpha-2}\left(1+\nu_{\mathrm{s}}\right) w+A q w^{\prime \prime}+\frac{\mathrm{i} \sigma C}{\Gamma} w^{\prime}-\eta \frac{D}{\Gamma} v^{\prime}=0 \\
-12 \eta \varepsilon^{2 \alpha-2} v^{\prime \prime}-12 \varepsilon^{3 \alpha-2}\left(1+\nu_{\mathrm{s}}\right) w^{\prime}+\eta \frac{\mathrm{i} \sigma C}{\Gamma} v^{\prime}+\frac{D}{\Gamma} w^{\prime}=0 .
\end{array}
$$

We still need to determine $\alpha$ and $\eta$. Depending on the value of $\alpha$, dominant balance in (5.19)-(5.20) will vary. We will first search for the distinguished limits [10]; i.e., 
TABLE 5.1

Significant terms in (5.19)-(5.20) for particular values of $\alpha$, with corresponding values of $\eta$. The terms in (5.19) are numbered $1-6$ and those in (5.20) are numbered 1-4.

\begin{tabular}{|r|c|c|c|c|c|}
\hline & $\alpha=\frac{1}{2}$ & $\alpha \in\left(\frac{1}{2}, \frac{2}{3}\right)$ & $\alpha=\frac{2}{3}$ & $\alpha \in\left(\frac{2}{3}, 1\right)$ & $\alpha=1$ \\
\hline$\eta=$ & $\varepsilon^{1 / 2}$ & $\varepsilon^{\alpha}$ & $\varepsilon^{2 / 3}$ & $\varepsilon^{2-2 \alpha}$ & 1 \\
\hline Terms kept in (5.19): & $1,2,3,4,5$ & $1,4,5$ & $1,4,5$ & $1,4,5$ & $1,4,5,6$ \\
\hline Terms kept in (5.20): & 1,2 & 1,2 & $1,2,4$ & 1,4 & $1,3,4$ \\
\hline
\end{tabular}

the specific values of $\alpha$ and $\eta$ for which four or more terms are balanced in (5.19), or for which three or more terms are balanced in (5.20).

The first distinguished limit is $\alpha=1 / 2$, which brings in the third term of (5.19) at leading order. In this case the second equation is dominated by the first two terms, implying $\eta=\varepsilon^{1 / 2}$.

The second distinguished limit is $\alpha=2 / 3$, which is obtained by balancing the second and fourth terms of (5.20). Balancing these with the first two terms gives $\eta=\varepsilon^{2 / 3}$. Finally the third distinguished limit is $\alpha=1, \eta=1$, which balances the first, third, and fourth terms in (5.20) and the first, fourth, fifth, and sixth terms in (5.19). We have therefore identified the three distinguished limits $\alpha=1 / 2, \alpha=2 / 3$, and $\alpha=$ 1 , which will henceforth be denoted as limits (i), (ii), and (iii), respectively. These are summarized in Table 5.1, where we have also included values of $\alpha$ in between the three distinguished limits, and chosen $\eta$ so that the required terms are significant. Now, for the first two distinguished limits, we will find the wavenumber of the oscillatory buckling pattern. The distinguished limit (iii), however, will be shown to be nonphysical, and we will not then analyze it further.

Before proceeding, we should determine the value of the constant $\sigma$. This came from the analysis of section 4 , so that if the displacement on the boundary of the half-plane was proportional to $\mathrm{e}^{\mathrm{i} k x}$, then $\sigma=\operatorname{sign}(k)$. In this case we have replaced the $x$-coordinate with the arc-length parameter $s=\widehat{R} \theta$, and we have $\mu=\cos \theta=$ $\sin (\pi / 2-\theta) \sim \pi / 2-\theta$ as we are close to the equator, $\theta=\pi / 2$. Thus we have

$$
\mathrm{e}^{\mathrm{i} \kappa \bar{\mu}}=\mathrm{e}^{\mathrm{i} \kappa \mu / \varepsilon^{\alpha}} \sim \exp \left(\frac{\mathrm{i} \kappa}{\varepsilon^{\alpha}}\left(\frac{\pi}{2}-\frac{s}{\widehat{R}}\right)\right) \propto \exp \left(-\frac{\mathrm{i} \kappa s}{\varepsilon^{\alpha} \widehat{R}}\right) .
$$

Comparing this with the $\mathrm{e}^{\mathrm{i} k x}$ used in the half-plane calculation, we find that $\sigma=$ $\operatorname{sign}\left(-\kappa / \varepsilon^{\alpha} \widehat{R}\right)$. If we limit ourselves without loss of generality to positive values of $\kappa$, we require $\sigma=-1$. This assumption will be propagated throughout the remainder of this article.

5.2.1. Distinguished limit (i). Here we have $\alpha=1 / 2$ and $\eta=\varepsilon^{1 / 2}$, giving, at leading order,

$$
\begin{aligned}
w^{\prime \prime \prime \prime}+12\left(1+\nu_{\mathrm{s}}\right) v^{\prime}+24\left(1+\nu_{\mathrm{s}}\right) w+A q w^{\prime \prime}-\frac{\mathrm{i} C}{\Gamma} w^{\prime} & =0, \\
v^{\prime \prime}+\left(1+\nu_{\mathrm{s}}\right) w^{\prime} & =0 .
\end{aligned}
$$

Seeking solutions of the form $w=a_{1} \mathrm{e}^{\mathrm{i} \kappa \bar{\mu}}, v=a_{2} \mathrm{e}^{\mathrm{i} \kappa \bar{\mu}}$ we find

$$
\kappa^{4}-A q \kappa^{2}+\frac{C}{\Gamma} \kappa+12\left(1-\nu_{\mathrm{s}}^{2}\right)=0
$$

Recall that this equation also gives us the leading-order critical stress $q$, simply by choosing the value of $q$ such that (5.24) has a double root. This is achieved by 
demanding that the derivative of the left-hand side of (5.24) with respect to $\kappa$ is also zero, so that $\kappa$ and $q$ are given by the simultaneous solution of (5.24) and $4 \kappa^{3}-2 A q \kappa+$ $\frac{C}{\Gamma}=0$. This second equation gives the leading-order critical stress in this case as

$$
q=\frac{1}{2 A \kappa}\left(4 \kappa^{3}+\frac{C}{\Gamma}\right)
$$

which can be substituted into (5.24) to find the quartic equation to be solved for $\kappa$ :

$$
\kappa^{4}-\frac{C \kappa}{2 \Gamma}-12\left(1-\nu_{\mathrm{s}}^{2}\right)=0
$$

5.2.2. Distinguished limit (ii). With $\alpha=2 / 3$ and $\eta=\varepsilon^{2 / 3}$, we substitute the oscillatory solution $w=a_{1} \mathrm{e}^{\mathrm{i} \kappa \bar{\mu}}, v=a_{2} \mathrm{e}^{\mathrm{i} \kappa \bar{\mu}}$ into the leading-order equation to find, by the same method, the leading-order critical stress

$$
q=\frac{3 \kappa^{2}}{A}, \quad \text { where } \quad \kappa=\left(\frac{C}{2 \Gamma}\right)^{1 / 3}
$$

5.2.3. Distinguished limit (iii). To understand why we do not consider this case, in which $\eta=1$ and $\alpha=1$, we remind ourselves that (2.1) was derived under the assumption that the wavelength $\lambda$ of the buckling pattern was small compared to the radius of curvature of the shell, and large compared to its thickness, i.e., $h \ll \lambda \ll \widehat{R}$. The wavelength in this case is given by $2 \pi k^{-1}$, where $k$ is the wavenumber identified in (5.21). Thus $\lambda=2 \pi \varepsilon^{\alpha} \widehat{R} / \kappa$. Hence, since $\varepsilon=h / \widehat{R},(2.1)$ is valid providing

$$
h \ll \frac{2 \pi \varepsilon^{\alpha} \widehat{R}}{\kappa} \ll \widehat{R}, \quad \text { i.e., } \quad \frac{h}{\widehat{R}} \ll \frac{2 \pi}{\kappa}\left(\frac{h}{\widehat{R}}\right)^{\alpha} \ll 1 .
$$

Thus we must have $0<\alpha<1$. The limit $\alpha=1$ corresponds to the case where the buckling wavelength is of the same order as the shell thickness. Equivalently, this restriction may be expressed in terms of the physical parameters of the problem,

$$
0<-\frac{\log \mathscr{G}}{3 \log \varepsilon}<1,
$$

by using (5.18) with $\Gamma=1$.

5.3. The envelope equation. In the previous section we obtained the oscillatory part of the buckling pattern, which was proportional to $\mathrm{e}^{\mathrm{i} \kappa \mu \varepsilon^{-\alpha}}$. The second step is to take this solution and multiply it by an envelope function. Accordingly we set

$$
w=\Re\left(f(\mu) \mathrm{e}^{\mathrm{i} \kappa \mu \varepsilon^{-\alpha}}\right), \quad v=\Re\left(g(\mu) \mathrm{e}^{\mathrm{i} \kappa \mu \varepsilon^{-\alpha}}\right) .
$$

These are substituted into the nondimensional Euler-Lagrange equations (5.12)(5.13), together with the aforementioned values of $\mathscr{G}, Q$, and $\sigma$. Furthermore, we make the scaling $\mu=\varepsilon^{\gamma} \widehat{\mu}$ for some $0<\gamma<\alpha$, and write the critical stress $q$ as an asymptotic expansion of the form $q=q_{0}+\varepsilon^{\beta} q_{1}+\cdots$, where $q_{0}$ is the leading-order critical stress obtained from sections 5.2.1-5.2.2 and $\beta>0$. With these assumptions, 
the largest terms in the equation are

$$
\begin{aligned}
& \varepsilon^{3 \alpha-4 \gamma} f^{\prime \prime \prime \prime}(\widehat{\mu})+4 \mathrm{i} \kappa \varepsilon^{2 \alpha-3 \gamma} f^{\prime \prime \prime}(\widehat{\mu})+\varepsilon^{\alpha-2 \gamma}\left(q_{0} A-6 \kappa^{2}\right) f^{\prime \prime}(\widehat{\mu}) \\
& +\varepsilon^{-\gamma}\left(2 \mathrm{i} \kappa q_{0} A-4 \mathrm{i} \kappa^{3}-\frac{\mathrm{i} C}{\Gamma}\right) f^{\prime}(\widehat{\mu})+\left[\varepsilon^{-\alpha} \kappa^{4}\left(1-2 \varepsilon^{2 \gamma} \widehat{\mu}^{2}\right)+24 \varepsilon^{3 \alpha-2}\left(1+\nu_{\mathrm{s}}\right)\right. \\
& \left.-\varepsilon^{-\alpha} \kappa^{2}\left(q_{0}+\varepsilon^{\beta} q_{1}\right)\left(A-\varepsilon^{2 \gamma}(B+A) \widehat{\mu}^{2}\right)+\frac{\kappa C}{\Gamma} \varepsilon^{-\alpha}\left(1-\frac{\varepsilon^{2 \gamma} \widehat{\mu}^{2}}{2}\right)\right] f(\widehat{\mu}) \\
& +\eta\left[12 \varepsilon^{3 \alpha-2-\gamma}\left(1+\nu_{\mathrm{s}}\right)-\varepsilon^{-\gamma} \frac{D}{\Gamma}\right] g^{\prime}(\widehat{\mu}) \\
& +\eta\left[12 \varepsilon^{2 \alpha-2}\left(1+\nu_{\mathrm{s}}\right) \mathrm{i} \kappa\left(1-\frac{\varepsilon^{2 \gamma} \widehat{\mu}^{2}}{2}\right)-\frac{\mathrm{i} \kappa D \varepsilon^{-\alpha}}{\Gamma}\left(1-\frac{\varepsilon^{2 \gamma} \widehat{\mu}^{2}}{2}\right)\right] g(\widehat{\mu})=0,
\end{aligned}
$$

$$
\begin{aligned}
& \text { (5.32) } \quad\left[-12 \varepsilon^{3 \alpha-2-\gamma}\left(1+\nu_{\mathrm{s}}\right)+\varepsilon^{-\gamma} \frac{D}{\Gamma}\right] f^{\prime}(\widehat{\mu}) \\
& +\left[-12 \varepsilon^{2 \alpha-2}\left(1+\nu_{\mathrm{s}}\right) \mathrm{i} \kappa\left(1-\frac{\varepsilon^{2 \gamma} \widehat{\mu}^{2}}{2}\right)+\frac{\varepsilon^{-\alpha} D \mathrm{i} \kappa}{\Gamma}\left(1-\frac{\varepsilon^{2 \gamma} \widehat{\mu}^{2}}{2}\right)\right] f(\widehat{\mu}) \\
& \quad-12 \eta \varepsilon^{3 \alpha-2-2 \gamma} g^{\prime \prime}(\widehat{\mu})+\eta\left[-24 \mathrm{i} \kappa \varepsilon^{2 \alpha-2-\gamma}-\frac{\varepsilon^{-\gamma} \mathrm{i} C}{\Gamma}\right] g^{\prime}(\widehat{\mu}) \\
& +\eta\left[12 \kappa^{2} \varepsilon^{\alpha-2}\left(1-\varepsilon^{2 \gamma} \widehat{\mu}^{2}\right)+12 \varepsilon^{3 \alpha-2}\left(1+\varepsilon^{2 \gamma} \widehat{\mu}^{2}\right)+\frac{C \kappa \varepsilon^{-\alpha}}{\Gamma}\left(1-\frac{\varepsilon^{2 \gamma} \widehat{\mu}^{2}}{2}\right)\right] g(\widehat{\mu})=0 .
\end{aligned}
$$

We now choose the appropriate values of $\beta$ and $\gamma$ for each of the distinguished limits identified earlier.

5.3.1. Distinguished limit (i). In this limit $\alpha=1 / 2$ and $\eta=\varepsilon^{1 / 2}$, and on examining (5.31) and (5.32), and substituting from (5.25) for $q_{0}$, we find that a suitable choice of indices is $\beta=1 / 2, \gamma=1 / 4$, whence

$$
\begin{gathered}
(5.33) \varepsilon^{-1 / 2}\left[\left(-\kappa^{4}+\frac{\kappa C}{2 \Gamma}+24\left(1+\nu_{\mathrm{s}}\right)\right) f(\widehat{\mu})+12\left(1+\nu_{\mathrm{s}}\right) \mathrm{i} \kappa g(\widehat{\mu})\right] \\
+\varepsilon^{-1 / 4} \cdot 12\left(1+\nu_{\mathrm{s}}\right) g^{\prime}(\widehat{\mu})+\left[\left(-4 \kappa^{2}+\frac{C}{2 \Gamma \kappa}\right) f^{\prime \prime}(\widehat{\mu})\right. \\
\left.+\left(\frac{\kappa B}{2 A}\left(4 \kappa^{3}+\frac{C}{\Gamma}\right) \widehat{\mu}^{2}-\kappa^{2} q_{1} A\right) f(\widehat{\mu})+\left(-6 \mathrm{i} \kappa\left(1+\nu_{\mathrm{s}}\right) \widehat{\mu}^{2}-\frac{\mathrm{i} \kappa D}{\Gamma}\right) g(\widehat{\mu})\right]+\cdots=0,
\end{gathered}
$$

$$
\begin{aligned}
& \varepsilon^{-1}\left(-12\left(1+\nu_{\mathrm{s}}\right) \mathrm{i} \kappa f(\widehat{\mu})+12 \kappa^{2} g(\widehat{\mu})\right)+\varepsilon^{-3 / 4}\left(-12\left(1+\nu_{\mathrm{s}}\right) f^{\prime}(\widehat{\mu})-24 \mathrm{i} \kappa g^{\prime}(\widehat{\mu})\right) \\
& \quad+\varepsilon^{-1 / 2}\left[-12 g^{\prime \prime}(\widehat{\mu})+\left(6\left(1+\nu_{\mathrm{s}}\right) \mathrm{i} \kappa \widehat{\mu}^{2}+\frac{D \mathrm{i} \kappa}{\Gamma}\right) f(\widehat{\mu})-12 \kappa^{2} \widehat{\mu}^{2} g(\widehat{\mu})\right]+\cdots=0 .
\end{aligned}
$$

Expanding $f(\widehat{\mu})$ and $g(\widehat{\mu})$ as $f \sim f_{0}+\varepsilon^{1 / 4} f_{1}+\varepsilon^{1 / 2} f_{2}+\cdots, g \sim g_{0}+\varepsilon^{1 / 4} g_{1}+\varepsilon^{1 / 2} g_{2}+\cdots$, we find that at leading order

$$
\begin{gathered}
\left(-\kappa^{4}+\frac{\kappa C}{2 \Gamma}+24\left(1+\nu_{\mathrm{s}}\right)\right) f_{0}(\widehat{\mu})+12\left(1+\nu_{\mathrm{s}}\right) \mathrm{i} \kappa g_{0}(\widehat{\mu})=0, \\
g_{0}(\widehat{\mu})=\frac{\left(1+\nu_{\mathrm{s}}\right) \mathrm{i}}{\kappa} f_{0}(\widehat{\mu}) .
\end{gathered}
$$

Copyright $@$ by SIAM. Unauthorized reproduction of this article is prohibited. 
As expected, using (5.26), these equations are linearly dependent. At the next order, substituting for $g_{0}(\widehat{\mu})$ from $(5.36)$, we obtain

$$
\begin{gathered}
\left(-\kappa^{4}+\frac{\kappa C}{2 \Gamma}+24\left(1+\nu_{\mathrm{s}}\right)\right) f_{1}(\widehat{\mu})+12\left(1+\nu_{\mathrm{s}}\right) \mathrm{i} \kappa g_{1}(\widehat{\mu})+\frac{12\left(1+\nu_{\mathrm{s}}\right)^{2} \mathrm{i}}{\kappa} f_{0}^{\prime}(\widehat{\mu})=0, \\
g_{1}(\widehat{\mu})=\frac{\left(1+\nu_{\mathrm{s}}\right) \mathrm{i}}{\kappa} f_{1}(\widehat{\mu})-\frac{\left(1+\nu_{\mathrm{s}}\right)}{\kappa^{2}} f_{0}^{\prime}(\widehat{\mu}) .
\end{gathered}
$$

On substituting for $g_{1}(\widehat{\mu})$ in (5.37), we again find that the equation is identically satisfied (as expected, since $\kappa$ is a double root of (5.26)). Finally, at third order, substituting for $g_{0}(\widehat{\mu})$ and $g_{1}(\widehat{\mu})$, we obtain

$$
\begin{gathered}
\left(-\kappa^{4}+\frac{\kappa C}{2 \Gamma}+24\left(1+\nu_{\mathrm{s}}\right)\right) f_{2}(\widehat{\mu})+12\left(1+\nu_{\mathrm{s}}\right) \mathrm{i} \kappa g_{2}(\widehat{\mu}) \\
+\frac{12 \mathrm{i}\left(1+\nu_{\mathrm{s}}\right)^{2}}{\kappa} f_{1}^{\prime}(\widehat{\mu})+\left(\frac{C}{2 \Gamma \kappa}-4 \kappa^{2}-\frac{12\left(1+\nu_{\mathrm{s}}\right)^{2}}{\kappa^{2}}\right) f_{0}^{\prime \prime}(\widehat{\mu}) \\
+\left[\frac{\kappa B}{2 A}\left(4 \kappa^{3}+\frac{C}{\Gamma}\right) \widehat{\mu}^{2}-\kappa^{2} q_{1} A+\left(1+\nu_{\mathrm{s}}\right)\left(6\left(1+\nu_{\mathrm{s}}\right) \widehat{\mu}^{2}+\frac{D}{\Gamma}\right)\right] f_{0}(\widehat{\mu})=0 \\
g_{2}(\widehat{\mu})=\frac{\left(1+\nu_{\mathrm{s}}\right) \mathrm{i}}{\kappa} f_{2}(\widehat{\mu})-\frac{\left(1+\nu_{\mathrm{s}}\right)}{\kappa^{2}} f_{1}^{\prime}(\widehat{\mu})-\frac{\mathrm{i}\left(1+\nu_{\mathrm{s}}\right)}{\kappa^{3}} f_{0}^{\prime \prime}(\widehat{\mu}) \\
+\frac{1}{12 \kappa^{2}}\left(6 \mathrm{i} \kappa\left(1+\nu_{\mathrm{s}}\right) \widehat{\mu}^{2}-\frac{D \mathrm{i} \kappa}{\Gamma}\right) f_{0}(\widehat{\mu}) .
\end{gathered}
$$

Substituting for $g_{2}(\widehat{\mu})$ in (5.39), and rearranging, we find

$$
f_{0}^{\prime \prime}(\widehat{\mu})+\left\{\left[\frac{\kappa^{2} q_{1} A-\frac{2\left(1+\nu_{\mathrm{s}}\right) D}{\Gamma}}{4 \kappa^{2}-\frac{C}{2 \Gamma \kappa}}\right]-\widehat{\mu}^{2}\left[\frac{\frac{\kappa B}{2 A}\left(4 \kappa^{3}+\frac{C}{\Gamma}\right)}{4 \kappa^{2}-\frac{C}{2 \Gamma \kappa}}\right]\right\} f_{0}(\widehat{\mu})=0 .
$$

A simple change of variables allows us to write the equation in the form $f_{0}^{\prime \prime}(\xi)+$ $\left(\Lambda-\xi^{2}\right) f_{0}(\xi)=0$, which is the harmonic oscillator problem seen earlier. As noted previously, the eigenvalues of this problem are the odd integers $\Lambda_{n}=(2 n+1)$, with corresponding eigenfunctions $f_{0}^{(n)}=H_{n}(\xi) \exp \left(-\frac{1}{2} \xi^{2}\right)$. The lowest stress at which the shells buckle corresponds to the lowest eigenvalue $\Lambda=1$. The corresponding eigenfunction is the Gaussian profile $f_{0}=\exp \left(-\frac{1}{2} \xi^{2}\right)$. Thus we obtain

$$
q_{1}=\frac{1}{A \kappa^{2}}\left\{\frac{2\left(1+\nu_{\mathrm{s}}\right) D}{\Gamma}+\left[\left(4 \kappa^{2}-\frac{C}{2 \Gamma \kappa}\right) \frac{\kappa B}{2 A}\left(4 \kappa^{3}+\frac{C}{\Gamma}\right)\right]^{1 / 2}\right\},
$$

and the corresponding envelope is given by

$$
f_{0}(\widehat{\mu})=\exp \left(-\frac{\widehat{\mu}^{2}}{2} \sqrt{\frac{\kappa^{2} B\left(4 \Gamma \kappa^{3}+C\right)}{A\left(8 \Gamma \kappa^{3}-C\right)}}\right),
$$

with $g_{0}(\widehat{\mu})$ given by $(5.36)$. 
In summary, returning to dimensional variables, we have

$$
\begin{aligned}
q \sim G_{\mathrm{m}} & \left\{\frac{\varepsilon}{2 A \kappa}\left(4 \kappa^{3}+\frac{C}{\Gamma}\right)\right. \\
& \left.+\frac{\varepsilon^{3 / 2}}{A \kappa^{2}}\left(\frac{2\left(1+\nu_{\mathrm{s}}\right) D}{\Gamma}+\left[\left(4 \kappa^{3}-\frac{C}{2 \Gamma}\right) \frac{B}{2 A}\left(4 \kappa^{3}+\frac{C}{\Gamma}\right)\right]^{1 / 2}\right)\right\},
\end{aligned}
$$

$$
v=\Re\left[\frac{\mathrm{i} \varepsilon^{1 / 2}\left(1+\nu_{\mathrm{s}}\right)}{\kappa} \exp \left(\frac{\mathrm{i} \kappa \mu}{\varepsilon^{1 / 2}}\right) \exp \left(-\frac{\mu^{2}}{2 \varepsilon^{1 / 2}} \sqrt{\frac{\kappa^{2} B\left(4 \Gamma \kappa^{3}+C\right)}{A\left(8 \Gamma \kappa^{3}-C\right)}}\right)\right]+O\left(\varepsilon^{3 / 4}\right),
$$

where $\kappa$ is the positive real solution to (5.26), $\Gamma=\varepsilon^{3 / 2} \mathscr{G}$, and $\varepsilon, \mathscr{G}$, and $A-D$ are as defined in section 5.1.

5.3.2. Distinguished limit (ii). We now follow the same pattern for the second distinguished limit, for which $\alpha=2 / 3$ and $\eta=\varepsilon^{2 / 3}$. Examining (5.31) and (5.32) we find that a suitable choice of indices is $\beta=2 / 3$ and $\gamma=1 / 3$. Space constraints do not allow us to display the full derivation here; the process is exactly analogous to that for distinguished limit (i). In dimensional variables, we have

$$
\begin{gathered}
q \sim G_{\mathrm{m}}\left\{\frac{3 \varepsilon^{2 / 3} \kappa^{2}}{A}+\frac{3 \varepsilon^{4 / 3}}{A}\left[\sqrt{\frac{\kappa^{2} B}{A}}+\frac{8\left(1+\nu_{\mathrm{s}}\right)}{\kappa^{2}}-\frac{1}{36 \kappa^{2}}\left(12\left(1+\nu_{\mathrm{s}}\right)-\frac{D}{\Gamma}\right)^{2}\right]\right\}, \\
w=\Re\left[\exp \left(\frac{\mathrm{i} \kappa \mu}{\varepsilon^{2 / 3}}\right) \exp \left(-\frac{\mu^{2}}{2 \varepsilon^{2 / 3}} \sqrt{\frac{\kappa^{2} B}{A}}\right)\right]+O\left(\varepsilon^{1 / 3}\right), \\
v\left[\frac{\mathrm{i} \varepsilon^{2 / 3}}{\kappa}\left(1+\nu_{\mathrm{s}}-\frac{D}{12 \Gamma}\right) \exp \left(\frac{\mathrm{i} \kappa \mu}{\varepsilon^{2 / 3}}\right) \exp \left(-\frac{\mu^{2}}{2 \varepsilon^{2 / 3}} \sqrt{\frac{\kappa^{2} B}{A}}\right)\right]+O(\varepsilon),
\end{gathered}
$$

where $\kappa$ is given by (5.27b), $\Gamma=\varepsilon^{\mathscr{G}}$, and $\varepsilon, \mathscr{G}$, and $A-D$ are as defined in section 5.1.

5.3.3. Uniform approximation for $\boldsymbol{q}$. The results of sections 5.3.1-5.3.2 are valid only where their respective definitions of $\Gamma$ are $O(1)$. Denoting quantities pertaining to the first and second distinguished limits by subscripts 1,2 , respectively, we have $\Gamma_{1}=\varepsilon^{3 / 2} \mathscr{G}$ and $\Gamma_{2}=\varepsilon \mathscr{G}$, or $\Gamma_{1}=\varepsilon^{1 / 2} \Gamma_{2}$. Thus the critical buckling stresses for each distinguished limit should match in the sense that the asymptotic expansion of $q_{1}$ as $\Gamma_{1} \rightarrow 0$ should overlap with that of $q_{2}$ as $\Gamma_{2} \rightarrow \infty$. A uniform expansion, valid in the regions of validity of both $\Gamma_{1}, \Gamma_{2}$, can be obtained by adding the two asymptotic expansions (5.44) and (5.47), and subtracting the overlapping terms.

Analyzing (5.44) and (5.47) in the appropriate $\Gamma_{i}$-limits yields

$$
\begin{aligned}
\frac{q_{1}}{G_{\mathrm{m}}} \sim & \frac{3 C^{2 / 3}}{2^{2 / 3} A} \varepsilon \Gamma_{1}^{-2 / 3}+\frac{1}{2^{1 / 3} A C^{2 / 3}}\left[4\left(1+\nu_{\mathrm{s}}\right) D+3 C \sqrt{\frac{B}{A}}\right] \varepsilon^{3 / 2} \Gamma_{1}^{-1 / 3} \\
& +\frac{2^{2 / 3} 12\left(1-\nu_{\mathrm{s}}^{2}\right)}{A C^{2 / 3}} \varepsilon \Gamma_{1}^{2 / 3}+\frac{8\left(1-\nu_{\mathrm{s}}^{2}\right)}{A C^{2}} \\
& \times\left[3 C \sqrt{\frac{B}{A}}-8\left(1+\nu_{\mathrm{s}}\right) D\right] \Gamma_{1} \varepsilon^{3 / 2}+\cdots \quad \text { as } \Gamma_{1} \rightarrow 0,
\end{aligned}
$$

Copyright $\odot$ by SIAM. Unauthorized reproduction of this article is prohibited. 
$(5.51)$

$$
\begin{aligned}
\frac{q_{2}}{G_{\mathrm{m}}} \sim & \frac{12}{A}\left(\frac{2}{C}\right)^{2 / 3}\left(1-\nu_{\mathrm{s}}^{2}\right) \varepsilon^{4 / 3} \Gamma_{2}^{2 / 3}+\frac{3}{A}\left(\frac{2}{C}\right)^{2 / 3}\left[\frac{C}{2} \sqrt{\frac{B}{A}}+\frac{2}{3}\left(1+\nu_{\mathrm{s}}\right) D\right] \varepsilon^{4 / 3} \Gamma_{2}^{-1 / 3} \\
+ & \frac{3}{A}\left(\frac{C}{2}\right)^{2 / 3} \varepsilon^{2 / 3} \Gamma_{2}^{-2 / 3}-\frac{D^{2}}{12 A}\left(\frac{2}{C}\right)^{2 / 3} \varepsilon^{4 / 3} \Gamma_{2}^{-4 / 3}+\cdots \quad \text { as } \Gamma_{2} \rightarrow \infty .
\end{aligned}
$$

By scaling $\Gamma_{1}, \Gamma_{2}$ appropriately we see that the overlap terms are the first three terms in each expansion. Furthermore, in the expression (5.51) we have not omitted any smaller terms in $\Gamma_{2}$ from the expansion (5.47), so the fourth term is the result of subtracting the overlap from $q_{2}$ and dividing by $G_{\mathrm{m}}$. Thus the uniform expansion is $q_{\text {unif }}=q_{1}+q_{2}-$ [overlap $]$, or

$$
\begin{aligned}
q_{\mathrm{unif}} & \sim G_{\mathrm{m}}\left\{-\frac{D^{2}}{12 A}\left(\frac{2}{C}\right)^{2 / 3} \Gamma_{1}^{-4 / 3} \varepsilon^{2}+\frac{\varepsilon}{2 A \kappa_{1}}\left(4 \kappa_{1}^{3}+\frac{C}{\Gamma_{1}}\right)\right. \\
& \left.+\frac{\varepsilon^{3 / 2}}{A \kappa_{1}^{2}}\left(\frac{2\left(1+\nu_{\mathrm{s}}\right) D}{\Gamma_{1}}+\left[\left(4 \kappa_{1}^{3}-\frac{C}{2 \Gamma_{1}}\right) \frac{B}{2 A}\left(4 \kappa_{1}^{3}+\frac{C}{\Gamma_{1}}\right)\right]^{1 / 2}\right)+\cdots\right\}
\end{aligned}
$$

5.4. Comparison with numerical results. We will now proceed to discuss how the two asymptotic expressions (5.44)-(5.46) and (5.47)-(5.49) compare with the full numerical results as computed by the methods of [12]. We recall first that the choice of $\alpha$ is determined by the relative size of $\mathscr{G}=G_{\mathrm{s}} / G_{\mathrm{m}}$ and $\varepsilon=h / \widehat{R}$, in such a way that $\Gamma$ as defined by (5.18) is an $O(1)$ parameter. We, however, calculated only the solutions for two values of $\alpha$, namely $\alpha=1 / 2$ (for which $\Gamma=\mathscr{G} \varepsilon^{3 / 2}$ ) and $\alpha=2 / 3$ (for which $\Gamma=\mathscr{G} \varepsilon$ ).

To examine the accuracy of the asymptotic expressions for the two values of $\alpha$, we examine the buckling patterns in the limit as $\varepsilon \rightarrow 0$, where we assume that $\Gamma=1$ in each case. For $\alpha=1 / 2$, this corresponds to assuming that $\mathscr{G}=\varepsilon^{-3 / 2}$. The corresponding buckling patterns (5.45)-(5.46) are shown in Figure 5.1 for five values of $\varepsilon$. Similarly, for $\alpha=2 / 3$ we assume that $\mathscr{G}=\varepsilon^{-1}$. Again the buckling patterns (5.48)(5.49) are displayed in Figure 5.2 for five values of $\varepsilon$. The asymptotic results seem to
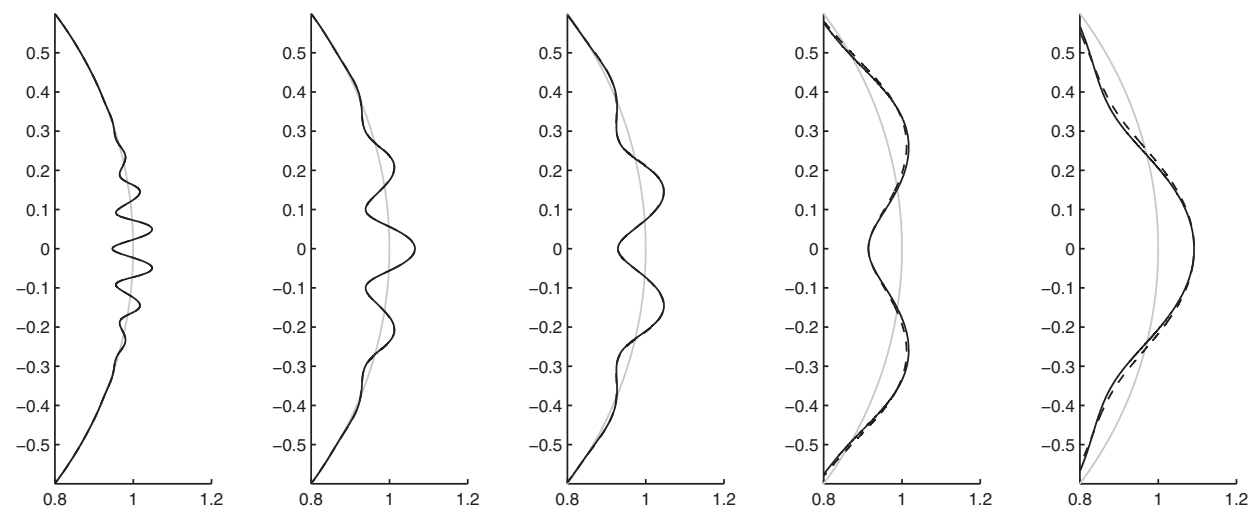

FIG. 5.1. The buckling patterns corresponding to the numerical solution of (2.1) (solid) compared with the asymptotic solution (5.45)-(5.46) (dashed), for $\mathscr{G}=\varepsilon^{-3 / 2}$ (i.e., $\alpha=1 / 2$ ) and $\varepsilon=0.001,0.005,0.01,0.05$, and 0.1 . 

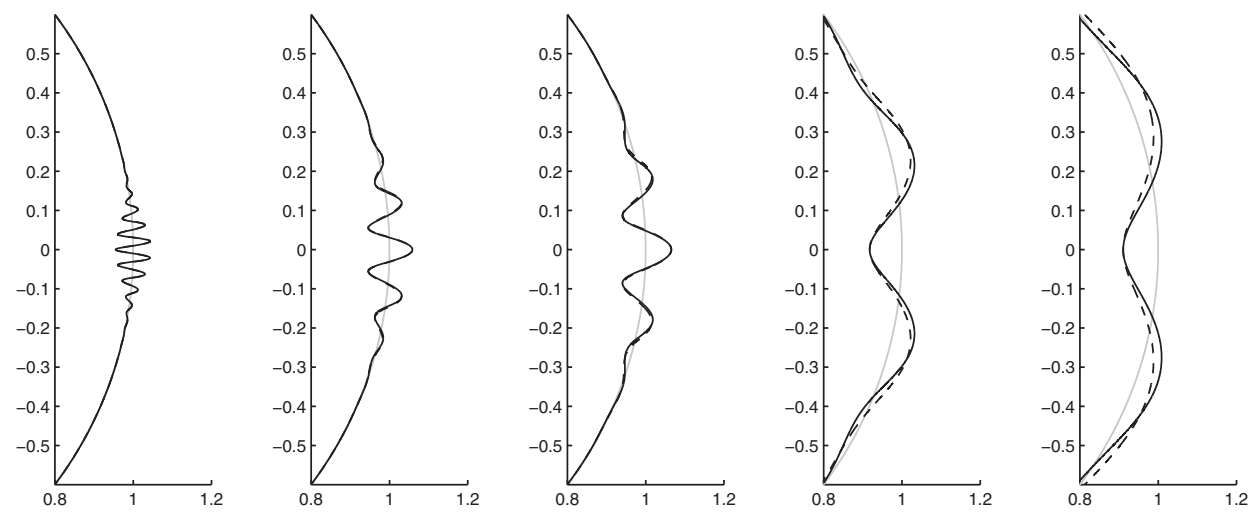

FIG. 5.2. The buckling patterns corresponding to the numerical solution of (2.1) (solid) compared with the asymptotic solution (5.48)-(5.49) (dashed), for $\mathscr{G}=\varepsilon^{-1}$ (i.e., $\alpha=2 / 3$ ) and $\varepsilon=0.001,0.005,0.01,0.05$, and 0.1 .

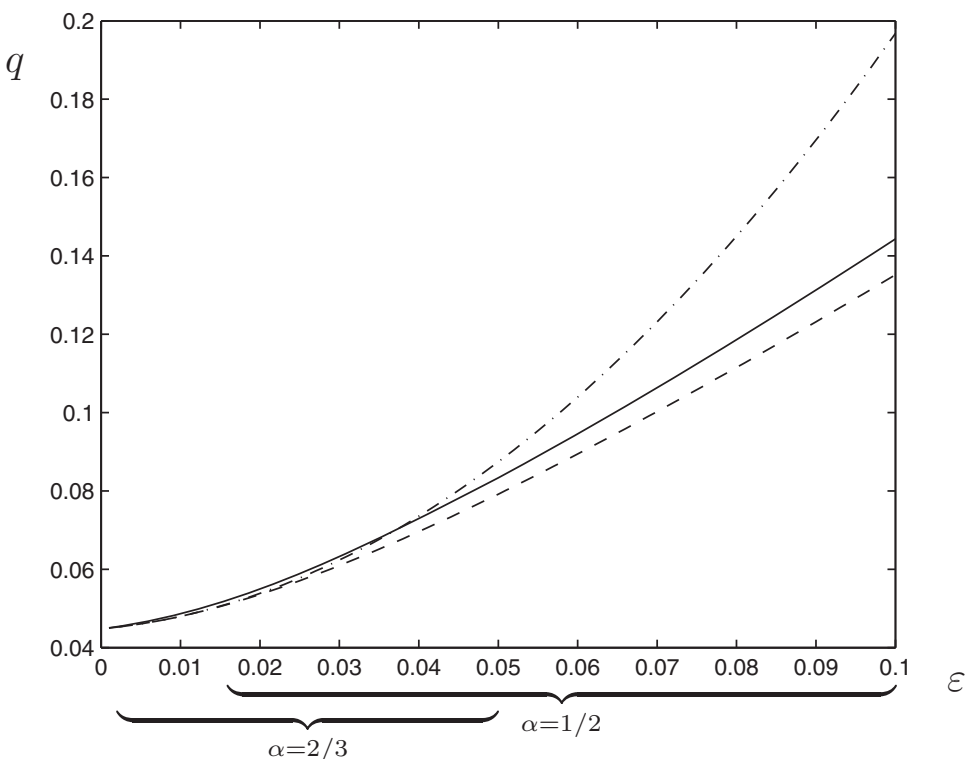

FIG. 5.3. A graph of the numerical solution to (2.1) (solid) compared with the asymptotic solution (5.44) (dashed) and (5.47) (dash-dot) as a function of $\varepsilon$ while keeping $\mathscr{G}=100$. At this resolution the uniform expansion (5.52) is indistinguishable from the dashed line.

agree well with the numerical results, especially comparing the buckling patterns for $\alpha=1 / 2$. One would not normally expect such a good agreement for $\varepsilon=0.1$ when the method assumes that $\varepsilon^{1 / 4} \approx 0.56$ is a small parameter.

Second, we will emulate the numerical experiments made in the introduction, whereby we vary $\varepsilon$ while keeping $\mathscr{G}$ constant, and vice versa. We will plot the solutions (5.44) and (5.47) corresponding to the two distinguished limits, and compare them to the full numerical solution. The thickness ratio $\varepsilon$ is first varied while keeping $\mathscr{G}=100$ constant, with the results shown in Figure 5.3. Assuming that $\Gamma$ is $O(1)$ if it lies in the interval $(0.2,5)$, we find that the $\alpha=1 / 2$ solution is valid for $\varepsilon \in\left(0.002^{2 / 3}, 0.05^{2 / 3}\right)$, 


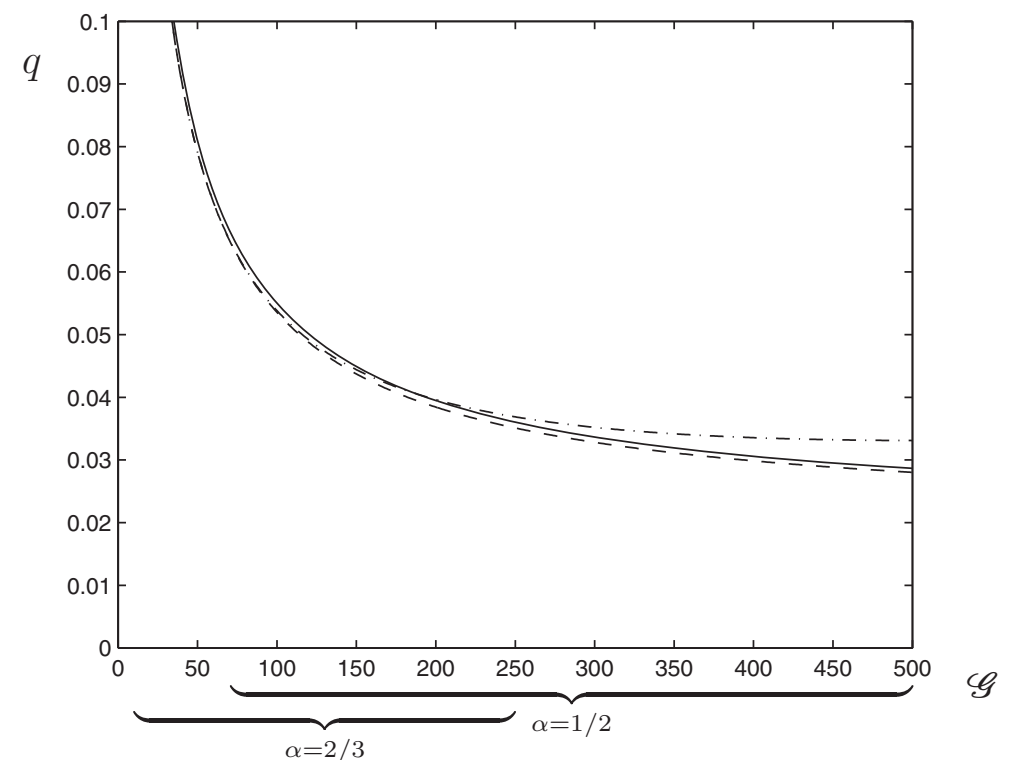

FIG. 5.4. A graph of the numerical solution to (2.1) (solid) compared with the asymptotic solution (5.44) (dashed) and (5.47) (dash-dot) as a function of $\mathscr{G}$ while keeping $\varepsilon=0.02$. At this resolution the uniform expansion (5.52) is indistinguishable from the dashed line.

and the $\alpha=2 / 3$ solution is valid for $\varepsilon \in(0.002,0.05)$. These regions are indicated on the graph. The same can be seen in Figure 5.4 for the case where we keep $\varepsilon=0.02$ constant and vary $\mathscr{G}$. The regions of validity of the two approximations are indicated on the graph. At the resolutions of these plots, the uniform expansion (5.52) - which is valid wherever either of the other two expressions are valid - is indistinguishable from the asymptotic solution (5.44), for which $\alpha=1 / 2$. The difference between the two becomes more noticeable near very small values of $\varepsilon$ or $\mathscr{G}$, where the $\alpha=2 / 3$ solution agrees better with the numerical solution.

6. Conclusions. In this article we have succeeded in analyzing asymptotically the buckling problem presented in [12], and in the process we have obtained very good approximations to the full solution, judging by the graphs obtained in section 5.4. Of particular surprise was the effectiveness of the asymptotic solution in the case $\alpha=1 / 2$, and the agreement between the asymptotic and numerical buckling patterns, of which the former is predicated on $\varepsilon^{1 / 4}$ being a small parameter.

In the process of obtaining these solutions we obtained an asymptotically valid solution for the stress on a half-plane when the displacements are amplitude-modulated oscillatory functions, and we believe that this solution may be of interest to researchers examining other similar buckling problems which involve a deformable substrate.

Prior to this analysis, we had no way of determining a priori whether the problem associated with the minimization of (2.1) was valid, in the sense that the buckling wavelength must be no larger than the shell radius and no smaller than the shell thickness. By showing that the parameter $\alpha$ - obtained by ensuring that $\Gamma$ is $O(1)$ must be between 0 and 1, or equivalently by satisfying (5.29), we can now determine whether a solution is physically valid before investing the time in minimizing (2.1) numerically. 
Of course, even with the restriction above on $\alpha$, the solution to (2.1) can not be considered to be the exact solution. Small imperfections in the shell would most likely cause it to buckle at a lower applied stress. However this does not discount the results that we have obtained here as they may be considered to be upper bounds to the true solution.

As stated in the introduction, this article considers only axisymmetric buckling deformations, in accordance with the hypothesis of the original paper [12]. It is not immediately clear that the axisymmetric mode is the critical one. Indeed, many investigations of the buckling of thin structures (for example, [5, 17]) have concluded that inherently two-dimensional buckling patterns are observed. However, in contrast to those situations, the pre-buckling stress distribution in the shell in our investigation is quite anisotropic, as stated in [12]. Both hoop stresses are at their most negative at the equator (in compression) but the magnitude of the latitudinal pre-buckling stress $\tau_{\theta \theta}^{\text {pre }}$ is much greater than that of the azimuthal stress $\tau_{\phi \phi}^{\text {pre }}$. As such the stretching energy that would be relieved by nonaxisymmetric buckling would not be sufficient to compensate for the additional bending energy induced. This conjecture is confirmed by a full nonaxisymmetric numerical investigation, to be published elsewhere.

Even if nonaxisymmetric deformations are included, the theory presented here is still a much-simplified model of the embedded-sphere problem. Undoubtedly, subtle effects at the shell-matrix interface could have a significant effect on the buckling behavior. It may be worth investigating the bonding mechanism to evaluate whether the critical buckling stress is greatly affected. A fully-debonded shell would most likely experience a different buckling pattern; the greater compliance of the matrix means that it would lose contact with the shell except at the poles, so that the deformation is reminiscent of sandwiching a sphere between two flat plates, for which buckling preferentially occurs at the poles rather than the equator. A bonding mechanism between the extremes of fully-bonded and debonded could have interesting effects, but would also make the post-buckling analysis much more difficult, due to the need to consider a coupled shell bonding and delamination problem. Moreover, it is not clear that the oscillatory pattern that facilitated the analysis is this article would also occur in the unbonded or partly-bonded cases.

Acknowledgment. The authors would like to thank the referees for their useful and constructive comments.

\section{REFERENCES}

[1] C.M. Bender and S.A. Orszag, Advanced Mathematical Methods for Scientists and Engineers, Springer, New York, 1999.

[2] P. Bunn And J.T. Mottram, Manufacture and compression properties of syntactic foams, Composites, 24 (1993), pp. 565-571.

[3] E. Cerda and L. Mahadevan, Geometry and physics of wrinkling, Phys. Rev. Lett., 90 (2003), p. 074302.

[4] S.J. Chapman, J.M.H. Lawry, J.R. Ockendon, and R.H. Tew, On the theory of complex rays, SIAM Rev., 41 (1999), pp. 417-509.

[5] X. Chen and J.W. Hutchinson, Herringbone buckling patterns of compressed thin films on compliant substrates, J. Appl. Mech., 71 (2004), pp. 597-603.

[6] C.D. Coman, Inhomogeneities and localised buckling patterns, IMA J. Appl. Math., 71 (2006), pp. 133-152.

[7] J. Genzer and J. Groenewold, Soft matter with hard skin: From skin wrinkles to templating and material characterization, Soft Matter, 2 (2006), pp. 310-323.

[8] M.E. Gurtin And E. Sternberg, Theorems in linear elastostatics for exterior domains, Arch. Ration. Mech. Anal., 8 (1961), pp. 99-119. 
[9] M. HeténYi, Beams on Elastic Foundation, University of Michigan Press, Ann Arbor, MI, 1946.

[10] E.J. Hinch, Perturbation Methods, Cambridge University Press, London, 1991.

[11] G.W. Hunt, M.K. Wadee, And N. Shiacolas, Localized elasticae for the strut problem on the linear foundation, J. Appl. Mech., 60 (1993), pp. 1033-1038.

[12] G.W. Jones, S.J. Chapman, And D.J. Allwright, Axisymmetric buckling of a spherical shell embedded in an elastic medium under uniaxial stress at infinity, Quart. J. Mech. Appl. Math., 61 (2008), pp. 475-495.

[13] W.T. KoITER, The nonlinear buckling problem of a complete spherical shell under uniform external pressure, P. K. Ned. Akad. B Phys., 72 (1969), pp. 40-123.

[14] S.H. LeE AND A.M. WAAS, Initial post-buckling behavior of a finite beam on an elastic foundation, Int. J. Non Lin. Mech., 31 (1996), pp. 313-328.

[15] M. Porfiri AND N. Gupta, Effect of volume fraction and wall thickness on the elastic properties of hollow particle filled composites, Compos. B Eng., 40 (2009), pp. 166-173.

[16] R.A. Ruhno and B.W. Sands, Hollow spheres, in Handbook of Fillers for Plastics, H. Katz and J. Milewski, eds., Springer, New York, 1987, Chap. 22.

[17] A. Vaziri and L. Mahadevan, Localized and extended deformations of elastic shells, Proc. Natl. Acad. Sci. USA, 105 (2008), pp. 7913-7918.

Copyright (c) by SIAM. Unauthorized reproduction of this article is prohibited. 